\title{
FAUST. II. Discovery of a Secondary Outflow in IRAS 15398-3359: Variability in Outflow Direction during the Earliest Stage of Star Formation?
}

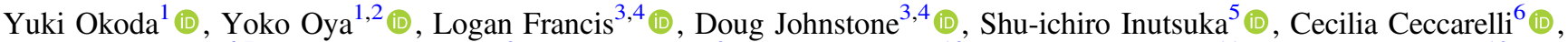

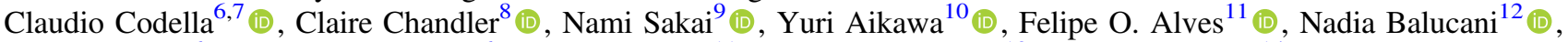
Eleonora Bianchi ${ }^{6}$ (1) , Mathilde Bouvier ${ }^{6}$ (1), Paola Caselli ${ }^{11}$ (1), Emmanuel Caux ${ }^{13}$, Steven Charnley ${ }^{14}$, Spandan Choudhury ${ }^{11}$, Marta De Simone $^{6}$ (1) , Francois Dulieu $^{15}$, Aurora Durán ${ }^{16}$, Lucy Evans ${ }^{7,13}$, Cécile Favre ${ }^{6}$, Davide Fedele ${ }^{7,17}$, Siyi Feng ${ }^{18,19,20}$ (1),

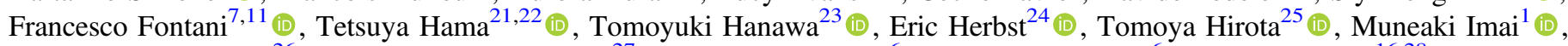

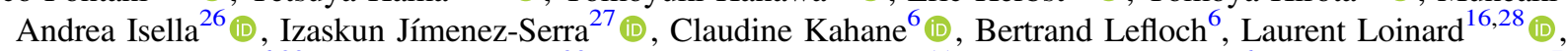

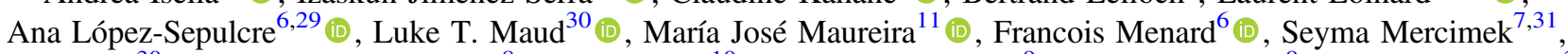
Anna Miotello $^{30}{ }^{(1)}$, George Moellenbrock ${ }^{8}$ (i), Shoji Mori ${ }^{10}$ (1), Nadia M. Murillo ${ }^{9}$, Riouhei Nakatani ${ }^{9}$ (i), Hideko Nomura ${ }^{32}$ (1),

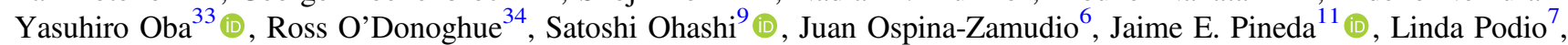
Albert Rimola $^{35}$ (1) , Takeshi Sakai ${ }^{36}$ (1) , Dominique Segura-Cox ${ }^{11}$ (1) , Yancy Shirley ${ }^{37}$, Brian Svoboda ${ }^{8,39}$ (i), Vianney Taquet ${ }^{7}$ (1) Leonardo Testi ${ }^{7,30}$ (10), Charlotte Vastel ${ }^{13}$, Serena Viti ${ }^{34}$ (1), Naoki Watanabe ${ }^{33}$ (1) , Yoshimasa Watanabe $^{38}$ (1), Arezu Witzel ${ }^{6}$, Ci Xue ${ }^{24}$ (1) , Yichen Zhang ${ }^{9}$ (i), Bo Zhao ${ }^{11}$, and Satoshi Yamamoto ${ }^{1,2}$

${ }^{1}$ Department of Physics, The University of Tokyo, 7-3-1, Hongo, Bunkyo-ku, Tokyo 113-0033, Japan; okoda@taurus.phys.s.u-tokyo.ac.jp

${ }^{2}$ Research Center for the Early Universe, The University of Tokyo, 7-3-1, Hongo, Bunkyo-ku, Tokyo 113-0033, Japan

${ }^{3}$ NRC Herzberg Astronomy and Astrophysics, 5071 West Saanich Road, Victoria, BC, V9E 2E7, Canada

${ }^{4}$ Department of Physics and Astronomy, University of Victoria, Victoria, BC, V8P 5C2, Canada

${ }^{5}$ Department of Physics, Nagoya University, Furo-cho, Chikusa-ku, Nagoya, Aichi 464-8602, Japan

${ }^{6}$ Univ. Grenoble Alpes, CNRS, IPAG, F-38000 Grenoble, France

${ }^{7}$ INAF, Osservatorio Astrofisico di Arcetri, Largo E. Fermi 5, I-50125, Firenze, Italy

${ }^{8}$ National Radio Astronomy Observatory, PO Box O, Socorro, NM 87801, USA

${ }^{9}$ RIKEN Cluster for Pioneering Research, 2-1, Hirosawa, Wako-shi, Saitama 351-0198, Japan

${ }^{10}$ Department of Astronomy, The University of Tokyo, 7-3-1 Hongo, Bunkyo-ku, Tokyo 113-0033, Japan

${ }^{11}$ Center for Astrochemical Studies, Max-Planck-Institut für extraterrestrische Physik (MPE), Gießenbachstr. 1, D-85741 Garching, Germany

${ }^{12}$ Department of Chemistry, Biology, and Biotechnology, The University of Perugia, Via Elce di Sotto 8, I-06123 Perugia, Italy

${ }^{13}$ IRAP, Université de Toulouse, CNRS, CNES, UPS, Toulouse, France

${ }_{14}^{14}$ Astrochemistry Laboratory, Code 691, NASA Goddard Space Flight Center, 8800 Greenbelt Road, Greenbelt, MD 20771, USA

${ }_{15}^{15}$ CY Cergy Paris Université, Sorbonne Université, Observatoire de Paris, PSL University, CNRS, LERMA, F-95000, Cergy, France

${ }^{16}$ Instituto de Radioastronomía y Astrofísica, Universidad Nacional Autónoma de México, A.P. 3-72 (Xangari), 8701, Morelia, Mexico

${ }^{17}$ INAF, Osservatorio Astrofisico di Torino, Via Osservatorio 20, 10025, Pino Torinese, Italy

${ }^{18}$ CAS Key Laboratory of FAST, National Astronomical Observatory of China, Datun Road 20, Chaoyang, Beijing, 100012, People's Republic of China

${ }_{20}^{19}$ National Astronomical Observatory of Japan, National Institutes of Natural Sciences, 2-21-1 Osawa, Mitaka, Tokyo 181-8588, Japan

${ }^{20}$ Academia Sinica Institute of Astronomy and Astrophysics, No.1, Sec. 4, Roosevelt Road, Taipei 10617, Taiwan, Republic of China

${ }_{21}^{21}$ Komaba Institute for Science, The University of Tokyo, 3-8-1 Komaba, Meguro, Tokyo 153-8902, Japan

${ }^{22}$ Department of Basic Science, The University of Tokyo, 3-8-1 Komaba, Meguro, Tokyo 153-8902, Japan

${ }^{23}$ Center for Frontier Science, Chiba University, 1-33 Yayoi-cho, Inage-ku, Chiba 263-8522, Japan

${ }^{24}$ Department of Chemistry, University of Virginia, McCormick Road, PO Box 400319, Charlottesville, VA 22904, USA

${ }^{25}$ National Astronomical Observatory of Japan, Osawa 2-21-1, Mitaka-shi, Tokyo 181-8588, Japan

${ }^{26}$ Department of Physics and Astronomy, Rice University, 6100 Main Street, MS-108, Houston, TX 77005, USA

${ }^{27}$ Centro de Astrobiología (CSIC-INTA), Ctra. de Torrejón a Ajalvir, km 4, E-28850, Torrejón de Ardoz, Spain

${ }^{28}$ Instituto de Astronomía, Universidad Nacional Autónoma de México, Ciudad Universitaria, A.P. 70-264, Cuidad de México 04510, Mexico

${ }^{29}$ Institut de Radioastronomie Millimétrique, F-38406 Saint-Martin d'Hères, France

${ }^{30}$ European Southern Observatory, Karl-Schwarzschild Str. 2, D-85748 Garching bei München, Germany

${ }^{31}$ Università degli Studi di Firenze, Dipartimento di Fisica e Astronomia, via G. Sansone 1, I-50019 Sesto Fiorentino, Italy

${ }_{32}$ Division of Science, National Astronomical Observatory of Japan, 2-21-1 Osawa, Mitaka, Tokyo 181-8588, Japan

${ }^{33}$ Institute of Low Temperature Science, Hokkaido University, N19W8, Kita-ku, Sapporo, Hokkaido 060-0819, Japan

${ }^{34}$ Department of Physics and Astronomy, University College London, Gower Street, London WC1E 6BT, UK

${ }^{35}$ Departament de Química, Universitat Autònoma de Barcelona, E-08193 Bellaterra, Spain

${ }^{36}$ Graduate School of Informatics and Engineering, The University of Electro-Communications, Chofu, Tokyo 182-8585, Japan ${ }^{37}$ Steward Observatory, 933 N Cherry Ave., Tucson, AZ 85721, USA

${ }^{38}$ Materials Science and Engineering, College of Engineering, Shibaura Institute of Technology, 3-7-5 Toyosu, Koto-ku, Tokyo 135-8548, Japan Received 2020 October 26; revised 2021 January 15; accepted 2021 January 18; published 2021 March 22

\begin{abstract}
We have observed the very low-mass Class 0 protostar IRAS 15398-3359 at scales ranging from 50 to 1800 au, as part of the Atacama Large Millimeter/Submillimeter Array Large Program FAUST. We uncover a linear feature, visible in $\mathrm{H}_{2} \mathrm{CO}$, $\mathrm{SO}$, and $\mathrm{C}^{18} \mathrm{O}$ line emission, which extends from the source in a direction almost perpendicular to the known active outflow. Molecular line emission from $\mathrm{H}_{2} \mathrm{CO}, \mathrm{SO}, \mathrm{SiO}$, and $\mathrm{CH}_{3} \mathrm{OH}$ further reveals an arc-like structure connected to the outer end of the linear feature and separated from the protostar, IRAS 15398-3359, by 1200 au. The arc-like structure is blueshifted with respect to the systemic velocity. A velocity gradient of $1.2 \mathrm{~km} \mathrm{~s}^{-1}$ over $1200 \mathrm{au}$ along the linear feature seen in the $\mathrm{H}_{2} \mathrm{CO}$ emission connects the protostar and the arc-like structure
\end{abstract}

$\overline{39}$ Jansky Fellow of the National Radio Astronomy Observatory. 
kinematically. $\mathrm{SO}, \mathrm{SiO}$, and $\mathrm{CH}_{3} \mathrm{OH}$ are known to trace shocks, and we interpret the arc-like structure as a relic shock region produced by an outflow previously launched by IRAS $15398-3359$. The velocity gradient along the linear structure can be explained as relic outflow motion. The origins of the newly observed arc-like structure and extended linear feature are discussed in relation to turbulent motions within the protostellar core and episodic accretion events during the earliest stage of protostellar evolution.

Unified Astronomy Thesaurus concepts: Stellar jets (1607); Star formation (1569); Shocks (2086); Protostars (1302); Young stellar objects (1834); Interstellar medium (847); Interstellar molecules (849); Astrochemistry (75);

\section{Introduction}

Millimeter/submillimeter-wave observations are revealing the complex physical and chemical nature of low-mass protostellar systems during their earliest evolutionary stage. For instance, protostellar accretion bursts resulting in a large instantaneous increase in the protostellar luminosity and subsequent heating of the protostellar envelope have been suggested for Class 0 sources based on the observed spatial distribution of molecular species (Jørgensen et al. 2013; Hsieh et al. 2018, 2019). Furthermore, complex structure, consisting of arc-like features and dense clumps, has been reported around the very young protostellar core, L1521F (Tokuda et al. 2014; Favre et al. 2020), and for the first hydrostatic core candidate, Chamaeleon-MMS1 (Busch et al. 2020). Because the protostars are deeply embedded in their parent cores, interactions between a protostellar outflow and surrounding gas may contribute to such complicated morphologies. Thus, detailing these structures will provide us with an important clue to elucidating a dynamic feature in the earliest stage of protostellar evolution. Given these circumstances, it is increasingly important to explore carefully and in detail the earliest stage of star formation for specific sources.

IRAS $15398-3359$ is a young low-mass Class 0 protostellar source $\left(T_{\mathrm{bol}}=44 \mathrm{~K}\right.$; Jørgensen et al. 2013) located in the Lupus 1 molecular cloud ( $d=156$ pc; Dzib et al. 2018). A molecular outflow driven from the protostar has been found via singledish observations in CO emission (Tachihara et al. 1996; van Kempen et al. 2009). A variety of unsaturated carbon-chain molecules such as $\mathrm{CCH}, \mathrm{C}_{4} \mathrm{H}$, and $\mathrm{CH}_{3} \mathrm{CCH}$ are abundant on scales of a few thousand au around the protostar. Hence, this source is classified as a warm carbon-chain chemistry source (Sakai et al. 2009; Sakai \& Yamamoto 2013).

High-resolution observations with the Atacama Large Millimeter/Submillimeter Array (ALMA) toward this source have revealed the outflow structure and the disk/envelope system. A bipolar outflow extending from the northeast to the southwest (position angle, P.A. $220^{\circ}$ ) was reported in $\mathrm{CCH}$ and $\mathrm{H}_{2} \mathrm{CO}$ line emission by Oya et al. (2014). The authors evaluated the inclination angle of the outflow axis to be $70^{\circ}$ $\left(0^{\circ}\right.$ for a pole-on configuration) based on their outflow model. Thus, the outflow is launched almost parallel to the plane of the sky. Submillimeter Array (SMA) observations of CO emission confirm this outflow feature (Bjerkeli et al. 2016b). The outflow dynamical timescale was derived to be very short, $10^{2}-10^{3}$ yr (Oya et al. 2014; Y1ldiz et al. 2015; Bjerkeli et al. 2016b). Significantly, this protostar has a very low dynamical mass. Oya et al. (2014) and Yen et al. (2017) derived the upper limit of the protostellar mass to be $0.09 M_{\odot}$ and $0.01 M_{\odot}$ using the observed velocity structure of the $\mathrm{H}_{2} \mathrm{CO}$ and $\mathrm{C}^{18} \mathrm{O}$ line emission, respectively, at a resolution of $0.15(\sim 80 \mathrm{au})$. More recently, a disk structure with Keplerian rotation was observed in SO line emission at a higher angular resolution ( 0 !" 2; $\sim 30 \mathrm{au}$ ) and the protostellar mass was re-evaluated to be
$0.007_{-0.003}^{+0.004} M_{\odot}$ (Okoda et al. 2018). The authors reported that the central velocity of the disk structure, $5.5 \mathrm{~km} \mathrm{~s}^{-1}$, is slightly shifted from the systemic velocity of the protostellar core, $\sim 5.2 \mathrm{~km} \mathrm{~s}^{-1}$ (Oya et al. 2014; Yen et al. 2017). They also estimated the disk mass to be between $0.006 M_{\odot}$ and $0.001 M_{\odot}$ from $1.2 \mathrm{~mm}$ dust continuum emission, assuming a dust temperature of $20 \mathrm{~K}$ and $100 \mathrm{~K}$, respectively. Despite the measurement uncertainties, the disk thus appears to have a lower mass than the mass of the protostar. Kristensen et al. (2012) and Jørgensen et al. (2013) previously reported that the envelope mass is $0.5-1.2 M_{\odot}$. Therefore, IRAS $15398-3359$ should be in the earliest stage of protostellar evolution.

Due to these unique physical and chemical characteristics, IRAS 15398-3359 was selected as a target of the ALMA Large Program FAUST (Fifty AU STudy of the chemistry in the disk/envelope system of solar-like protostars ${ }^{40}$ ). This program aims at revealing the physical and chemical structure of 13 nearby protostars $(d=137-235 \mathrm{pc})$, at scales from a few thousand au down to $50 \mathrm{au}$, by observing various molecular lines. In addition to lines of fundamental molecules (e.g., $\mathrm{C}^{18} \mathrm{O}$ and $\mathrm{H}_{2} \mathrm{CO}$ ) and complex organic molecules, some shock tracer molecules, such as $\mathrm{SO}, \mathrm{SiO}$, and $\mathrm{CH}_{3} \mathrm{OH}$, are included in this program to reveal possible interactions between the outflow and the ambient gas: these species are thought to be liberated from dust grains or to be produced in the gas phase within shocked regions.

Taking advantage of the chemical diagnostic power of FAUST obtained by observing these shock tracer lines with high sensitivity, we have found a secondary outflow feature launched from IRAS 15398-3359, which provides a novel insight into the earliest stage of protostellar evolution. In Section 2 we present the relevant observations. Next, in Section 3 we discuss the results of our spatial and spectral investigation. We analyze and discuss these findings in Sections 4 and 5, respectively, before concluding the paper in Section 6 .

\section{Observations}

Single-field observations for IRAS 15398-3359 were conducted between 2018 October and 2019 January as part of the ALMA Large Program FAUST. The parameters of the observations are summarized in Table 1. The molecular lines analyzed in the frequency range from 217 to $220 \mathrm{GHz}$ (Band 6) are listed in Table 2 . We used the $12 \mathrm{~m}$ array data from the two different configurations (C43-5 and C43-2 for sparse and compact configurations, respectively) and the $7 \mathrm{~m}$ array data of the Atacama Compact Array (ACA/Morita Array), combining these visibility data in the UV plane. In total, the baseline lengths range from 7.43 to $1310.74 \mathrm{~m}$. The adopted field center was taken to be $\left(\alpha_{2000}, \delta_{2000}\right)=\left(15^{\mathrm{h}} \cdot 43^{\mathrm{m}} \cdot 02^{\mathrm{s}} \cdot 242,-34^{\circ} 09^{\prime}\right.$ 06." 805), which is close to the protostellar position. The

\footnotetext{
${ }^{40}$ http://faust-alma.riken.jp
} 
Table 1

Observation Parameters

\begin{tabular}{|c|c|c|c|}
\hline Parameter & Band $6(\mathrm{C} 43-5)$ & Band $6(\mathrm{C} 43-2)$ & Band 6 (7 m Array of ACA) \\
\hline Observation date(s) & 2018 Nov 23 & 2019 Jan 6 & 2018 Oct 24,25 \\
\hline Time on source (minute) & 47.10 & 12.63 & $28.75,28.77$ \\
\hline Number of antennas & 45 & 47 & 10 \\
\hline Primary beamwidth (arcsec) & 26.7 & 26.7 & 45.8 \\
\hline Total bandwidth (GHz) & 0.059 & 0.059 & 0.062 \\
\hline Continuum bandwidth (GHz) & 1.875 & 1.875 & 2.000 \\
\hline Proj. baseline range $(\mathrm{m})$ & $15.03-1310.74$ & $12.66-425.02$ & $7.43-46.74$ \\
\hline Bandpass calibrator & J1427-4206 & $\mathrm{J} 1427-4206$ & $\mathrm{~J} 1229+0203, \mathrm{~J} 1427-4206$ \\
\hline Phase calibrator & J1626-2951 & $\mathrm{J} 1517-2422$ & $\mathrm{~J} 1517-2422$ \\
\hline Flux calibrator & $\mathrm{J} 1427-4206$ & $\mathrm{~J} 1427-4206$ & $\mathrm{~J} 1229+0203, \mathrm{~J} 1427-4206$ \\
\hline Pointing calibrator & $\mathrm{J} 1650-2943, \mathrm{~J} 1427-4206$ & $\mathrm{~J} 1427-4206$ & $\mathrm{~J} 1229+0203, \mathrm{~J} 1427-4206, \mathrm{~J} 1517-2422$ \\
\hline Resolution (arcsec) (P.A. (deg)) & $0.338 \times 0.280(63.9)$ & $1.140 \times 0.921(79.9)$ & $8.210 \times 4.780(69.2)$ \\
\hline rms (mJy beam ${ }^{-1}$ channel $^{-1}$ ) & 1.8 & 4.4 & 24.0 \\
\hline
\end{tabular}

backend correlator for the molecular line observations was set to a resolution of $122 \mathrm{kHz}$ and a bandwidth of $62.5 \mathrm{MHz}$. The data were reduced in Common Astronomy Software Applications (CASA) package 5.4.1 (McMullin et al. 2007) using a modified version of the ALMA calibration pipeline and an additional in-house calibration routine (G. Moellenbrock et al. 2021 , in preparation) to correct for the $T_{\text {sys }}$ and spectral line data normalization. ${ }^{41}$ Self-calibration was carried out using line-free continuum emission, for each configuration. The complex gain corrections derived from the self-calibration were then applied to all channels in the data, and the continuum model derived from the self-calibration was subtracted from the data to produce continuum-subtracted line data. A selfcalibration technique was also used to align both amplitudes and phases (i.e., positions) across the multiple configurations. Images were prepared by using the tclean task in CASA, where Briggs weighting with a robustness parameter of 0.5 was employed. The primary beam correction was applied to all the images presented in this paper. Since the maximum recoverable scales are 12 !" 8 , any structures extending more than that size could be resolved out. The rms noise levels are $2 \mathrm{mJy}$ beam ${ }^{-1}$ channel $^{-1}$ for $\mathrm{H}_{2} \mathrm{CO}, \mathrm{SiO}, \mathrm{CH}_{3} \mathrm{OH}$, and $\mathrm{C}^{18} \mathrm{O}$, and $3 \mathrm{mJy}$ beam $^{-1}$ channel $^{-1}$ for SO. The original synthesized beam sizes are summarized in Table 2. The uncertainty in the absolute flux density scale is estimated to be $10 \%$ (Francis et al. 2020).

\section{A Possible Secondary Outflow}

\subsection{Primary Outflow in the Northeast to Southwest Direction}

Figures 1(a) and 2(a) show the moment 0 maps of the $\mathrm{H}_{2} \mathrm{CO}$ and SO line emission, respectively. The outflow structure along the northeast to southwest axis (P.A. $220^{\circ}$ ) is seen in the $\mathrm{H}_{2} \mathrm{CO}$ emission, as reported previously (Oya et al. 2014; Bjerkeli et al. 2016b). Contrastingly, the SO emission is concentrated around the protostar (Okoda et al. 2018, 2020) with little emission within the outflow except for a localized knot (Blob D in Okoda et al. 2020) seen in the southwestern lobe, which could be formed by the impact of the outflow on ambient gas. The $\mathrm{CH}_{3} \mathrm{OH}$ and $\mathrm{C}^{18} \mathrm{O}$ line emission traces part of the outflow in Figures 2(b) and (c). The knot can also be seen in the $\mathrm{CH}_{3} \mathrm{OH}$ emission (see also Okoda et al. 2020). Along with these previously known structures, we have found an additional

\footnotetext{
$\overline{41}$ https://help.almascience.org/index.php?/Knowledgebase/Article/ View $/ 419$
}

spatial feature extending toward the southeast and northwest of IRAS $15398-3359$.

\subsection{Arc-like Structure in the Southeast Direction}

Part of the $\mathrm{H}_{2} \mathrm{CO}$ line emission and a majority of the SO line emission are extended in the southeastern direction (P.A. $140^{\circ}$ ), which is also close to the disk/envelope direction (P.A. $130^{\circ}$ ) of the IRAS 15398-3359 protostar reported by Oya et al. (2014), Yen et al. (2017), and Okoda et al. (2018). The $\mathrm{H}_{2} \mathrm{CO}$ emission appears to bend toward the south about $8^{\prime \prime}(\sim 1200 \mathrm{au})$ from the protostar. The moment 0 map of the SO emission shows an arc-like structure around the southeastern part (Figure 2(a)), where the northern tip of the arc corresponds to the bending point seen in the $\mathrm{H}_{2} \mathrm{CO}$ emission. Although the arc-like structure is near the edge of the field of view for the ALMA $12 \mathrm{~m}$ data, it lies within the field of view of ACA data. Hence, the observed structure is real.

Along with the $\mathrm{H}_{2} \mathrm{CO}$ and $\mathrm{SO}$ line emission, the $\mathrm{SiO}$ and $\mathrm{CH}_{3} \mathrm{OH}$ line emission is found to trace the arc-like structure around the southeastern part. In contrast to the $\mathrm{H}_{2} \mathrm{CO}$ and $\mathrm{SO}$ line emission, the $\mathrm{SiO}$ emission is not detected toward the protostar, and mainly traces the southeastern part of the arc-like structure (Figure 2(d)). These features are also apparent in the molecular line profiles (Figure 3) toward four positions within the arc-like structure and at the protostellar position, as indicated in Figure 2. The line width of the $\mathrm{SiO}$ emission is slightly broader at position $\mathrm{D}\left(0.9 \mathrm{~km} \mathrm{~s}^{-1}\right)$ than at positions $\mathrm{A}$, $\mathrm{B}$, and $\mathrm{C}\left(0.3-0.8 \mathrm{~km} \mathrm{~s}^{-1}\right)$. Faint emission from $\mathrm{CH}_{3} \mathrm{OH}$ is also detected in part of the arc-like structure in addition to the previously observed outflow cavity wall (Figure 2(b)). While the $\mathrm{CH}_{3} \mathrm{OH}$ emission looks faint in the moment 0 map, its spectrum is clearly detected in the arc-like structure, as revealed in Figure 3.

In Figure 4, we present the velocity channel maps of the $\mathrm{H}_{2} \mathrm{CO}$, SO, SiO, and $\mathrm{CH}_{3} \mathrm{OH}$ line emission from the $3.9 \mathrm{~km} \mathrm{~s}^{-1}$ panel to the $5.5 \mathrm{~km} \mathrm{~s}^{-1}$ panel, where the systemic velocity of the protostellar core is $5.2 \mathrm{~km} \mathrm{~s}^{-1}$ (Yen et al. 2017). The velocity channel maps reveal the arc-like structure. The $\mathrm{CH}_{3} \mathrm{OH}$ emission is seen clearly in the velocity maps at 4.7 and $5.1 \mathrm{~km} \mathrm{~s}^{-1}$. On the other hand, the arc-like structure is not evident in the $\mathrm{C}^{18} \mathrm{O}$ emission, as shown in the line profiles and the velocity channel maps (Figures 3 and $4(\mathrm{e})$ ). The $\mathrm{C}^{18} \mathrm{O}$ emission usually traces cold and dense clumps of gas with relatively high column density. Although marginal emission 
Table 2

List of Observed Lines ${ }^{\mathrm{a}}$

\begin{tabular}{|c|c|c|c|c|c|}
\hline Molecule & Transition & Frequency $(\mathrm{GHz})$ & $S \mu^{2}\left(D^{2}\right)$ & $E_{\mathrm{u}} k^{-1}(\mathrm{~K})$ & Beam Size \\
\hline $\mathrm{H}_{2} \mathrm{CO}^{\mathrm{b}}$ & $3_{0,3}-2_{0,2}$ & 218.2221920 & 16.308 & 21 & $0 ! \prime 35 \times 0$ "! $30\left(\right.$ P.A. $\left.69^{\circ}\right)$ \\
\hline $\mathrm{SO}^{\mathrm{c}}$ & $\mathrm{N}_{J}=5_{6}-4_{5}$ & 219.9494420 & 14.015 & 35 & 0 " $35 \times 0$ 0" $30\left(\right.$ P.A. $\left.65^{\circ}\right)$ \\
\hline $\mathrm{SiO}^{\mathrm{d}}$ & $5-4$ & 217.1049190 & 47.993 & 31 & $0 ! 35 \times 0$ "! $30\left(\right.$ P.A. $\left.68^{\circ}\right)$ \\
\hline $\mathrm{C}^{18} \mathrm{O}^{\mathrm{f}}$ & $2-1$ & 219.5603541 & 0.024399 & 16 & $0 "$ " $35 \times 0$ 0" $29\left(\right.$ P.A. $\left.65^{\circ}\right)$ \\
\hline
\end{tabular}

Notes.

a Line parameters are taken from CDMS (Endres et al. 2016). The beam size for each line is obtained from the observations.

b Müller \& Lewen (2017).

c Klaus et al. (1996).

d Müller et al. (2013)

e Xu et al. (2008).

${ }^{\mathrm{f}}$ Klapper et al. (2001).

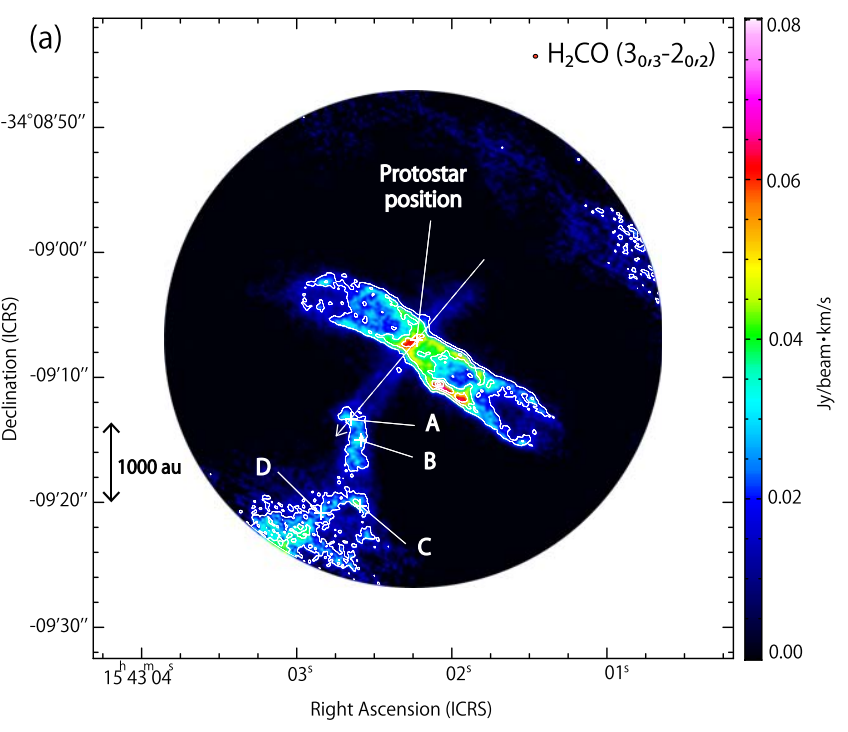

(b)

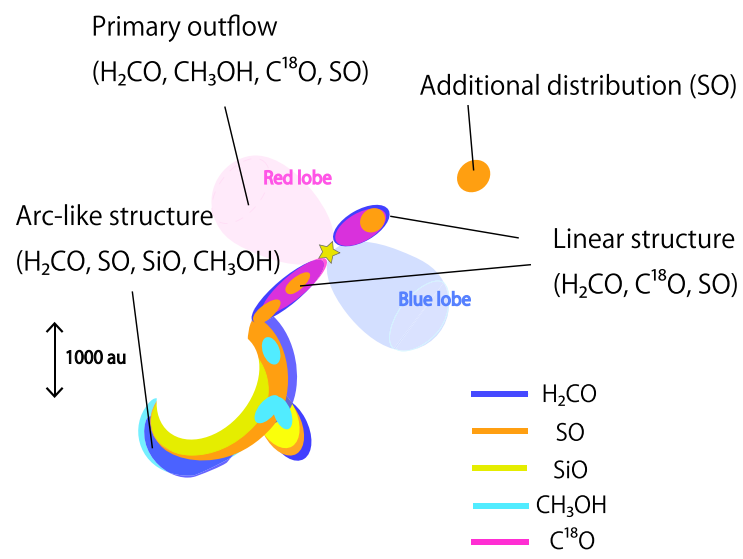

Figure 1. (a) Moment 0 map of the $\mathrm{H}_{2} \mathrm{CO}$ line emission. Contour levels are every $\sigma$ from $3 \sigma$, where $\sigma$ is $6 \mathrm{mJy}_{\text {beam }}{ }^{-1}$. The line intensities are integrated from 3.7 to $5.7 \mathrm{~km} \mathrm{~s}^{-1}$ to focus on the arc-like feature and linear structure. White cross marks show the five locations where molecular line profiles are produced (see Figure 3). The circle at top right represents the synthesized beam size. The dashed arrow represents the direction of the PV diagram. (b) Schematic picture of the molecular distributions.

may be detected around the southeastern extent in the map of $5.5 \mathrm{~km} \mathrm{~s}^{-1}$, the low $\mathrm{C}^{18} \mathrm{O}$ column density (calculated in Section 4.2) ensures that the arc-like structure is not part of an adjacent prestellar core.

\subsection{Shocks in the Arc-like Structure}

The $\mathrm{SO}, \mathrm{SiO}$, and $\mathrm{CH}_{3} \mathrm{OH}$ line emission are known to be enhanced in the gas phase by shocks and are often detected in active outflow-shocked regions (e.g., Bachiller \& Pérez Gutiérrez 1997), although $\mathrm{SO}$ and $\mathrm{CH}_{3} \mathrm{OH}$ are also present in quiescent starless cores (e.g., Spezzano et al. 2017; Punanova et al. 2018). A typical example of such a shock region in a lowmass star-forming region occurs in the outflow driven from the Class 0 protostar, IRAS 20386+6751, in L1157 (Mikami et al. 1992; Gueth et al. 1996; Bachiller \& Pérez Gutiérrez 1997). Outflows often produce shocked emission at locations where they interact with ambient gas. In these outflow-shocked regions, the chemical composition of the gas is drastically changed, mainly due to liberation of molecules from dust grains and subsequent gas-phase reactions. In L1157, the shocked region in the blueshifted outflow lobe (L1157-B1) is traced by $\mathrm{SO}, \mathrm{SiO}, \mathrm{CH}_{3} \mathrm{OH}, \mathrm{H}_{2} \mathrm{CO}, \mathrm{HCN}, \mathrm{CN}$, and $\mathrm{SO}_{2}$ line emission (Mikami et al. 1992; Gueth et al. 1996; Bachiller \& Pérez Gutiérrez 1997; Codella et al. 2010, 2020; Podio et al. 2017; Feng et al. 2020). Enhancement of the same molecules has also been reported for other protostellar outflows: e.g., L1448-C/L1448-mm (Jiménez-Serra et al. 2005; Hirano et al. 2010), HH211 (Hirano et al. 2006), NGCC1333-IRAS4A (Wakelam et al. 2005), and BHR71 (Gusdorf et al. 2015). Since $\mathrm{SiO}$ is a robust shock tracer, the observed arc-like structure to the southeast of IRAS 15398-3359 should also be a shocked region. We also detect enhancement in the abundance of other species, SO and $\mathrm{CH}_{3} \mathrm{OH}$ (Figures 2(a) and (b)), which is consistent with the enhancement reported toward the shocked region, L1157-B1. Furthermore, the morphology resembles a bow shock, somewhat similar to those revealed by the simulations (Smith et al. 1997; Lee et al. 2001) and the observation (HH46/47; Arce et al. 2013).

The line width of the $\mathrm{SiO}$ emission in typical shocked regions is generally broad $\left(\sim 10 \mathrm{~km} \mathrm{~s}^{-1}\right.$; e.g., Mikami et al. 1992; Bachiller \& Pérez Gutiérrez 1997). Here, however, the $\mathrm{SiO}$ emission has a narrow line width of about $1 \mathrm{~km} \mathrm{~s}^{-1}$ toward the arc-like structure (Figures 3 and 4(c)). The lack of a broad line width may be evidence that the shock is relatively old and that the turbulent motions produced within the shock region have dissipated. Such a fossil shock has been suggested as the explanation for similar observations of the sources HH7-11 (SVS13-A) and NGC 2264 by Codella et al. (1999) and LópezSepulcre et al. (2016), respectively. This hypothesis will be further discussed in relation to the origin of the arc-like 

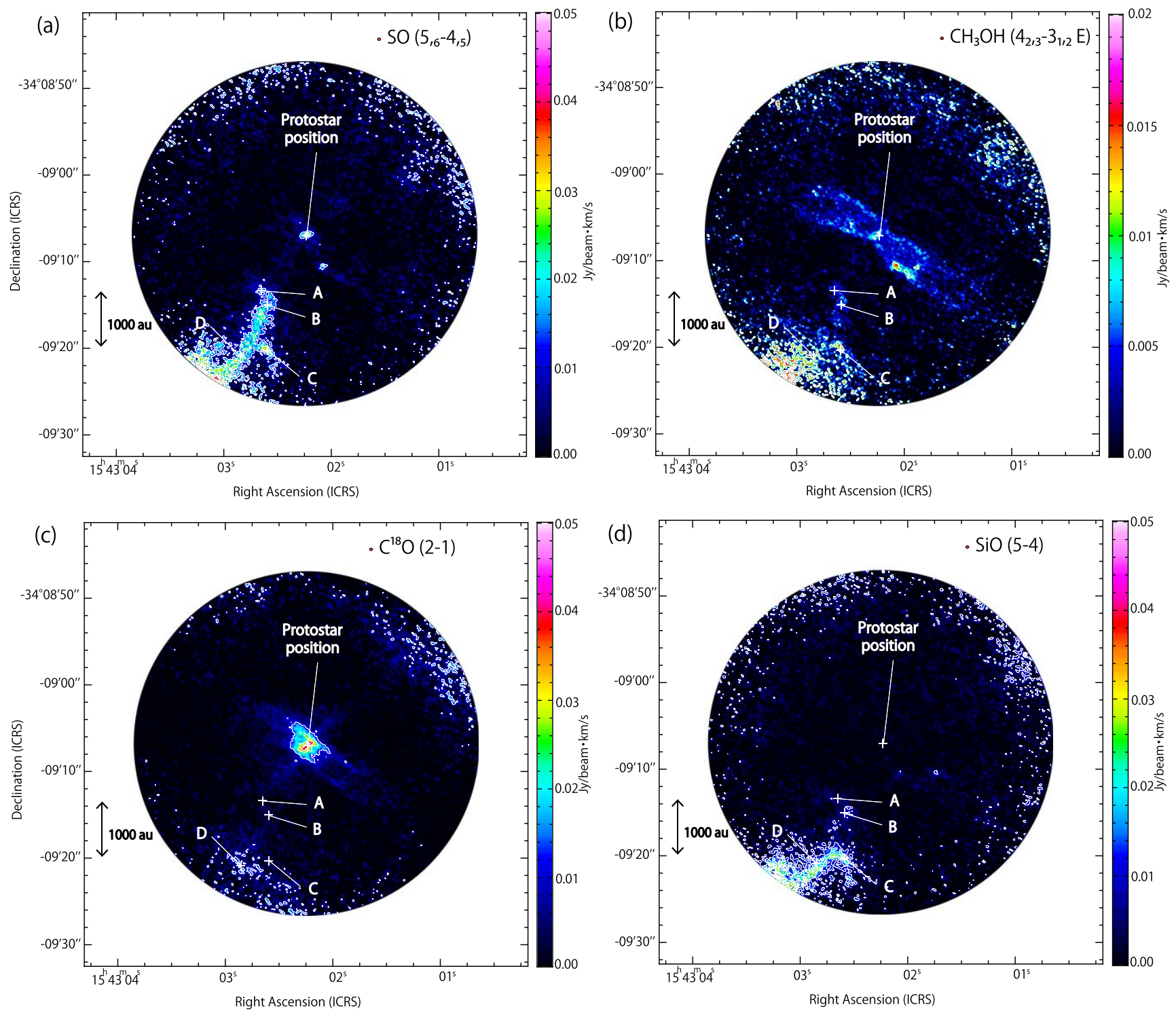

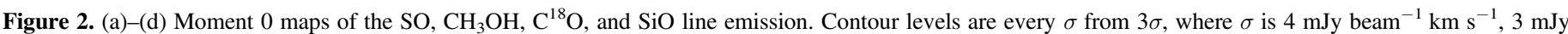
beam ${ }^{-1} \mathrm{~km} \mathrm{~s}^{-1}, 4 \mathrm{mJy}_{\text {beam }}^{-1} \mathrm{~km} \mathrm{~s}^{-1}$, and $3 \mathrm{mJy}_{\text {beam }}^{-1} \mathrm{~km} \mathrm{~s}^{-1}$, respectively. See Figure 1 caption for additional details.

structure in Section 5.2. We note that narrow line width of $\mathrm{SiO}$ can also be interpreted in terms of magnetohydrodynamic C-shocks (Jiménez-Serra et al. 2004, 2005, 2008, 2009).

IRAS 15398-3359 is located at the edge of the Lupus 1 molecular cloud, as can be seen in large-scale dust continuum and CO observations (Tothill et al. 2009; Gaczkowski et al. 2015; Mowat et al. 2017). A protostellar source has never been found through infrared observations (Rygl et al. 2013) toward the southeastern region of IRAS 15398-3359 where the arclike structure resides. Furthermore, submillimeter continuum emission is not detected at this location in our data sets, nor has ALMA previously uncovered continuum emission at this location (Jørgensen et al. 2013; Oya et al. 2014; Okoda et al. 2018). Combined with the previously mentioned lack of significant $\mathrm{C}^{18} \mathrm{O}$ emission, these null results suggest that there is no nearby enhancement of dense gas to the southeast of IRAS 15398-3359. Thus, the arc-like structure likely originates from the interaction of an outflow oriented along the northwest to southeast axis ejected from IRAS 15398 -3359 and its natal ambient gas. If this is the case, a detectable counter-lobe might be expected to the northwest of IRAS 15398-3359. A hint of this counter-lobe can be seen in the SO emission, as described in the next subsection.

\subsection{Linear Structure in the Northwest to Southeast Direction}

Focusing on the vicinity of the protostar, within $5^{\prime \prime}$, a linear structure is seen in the $\mathrm{H}_{2} \mathrm{CO}$ emission, connecting the southeast arc-like structure to the protostar and continuing toward the northwest (Figure 1(a)). This structure is more clearly identified in the velocity maps at 4.7 and $5.1 \mathrm{~km} \mathrm{~s}^{-1}$ (Figure 4(a)). Investigating this linear structure near the protostar, we find that the geometry is not simply an extension of the disk/envelope system related to the primary outflow (Figure 5). The linear structure is slightly inclined by about $10^{\circ}$ from the disk/envelope axis (P.A. $130^{\circ}$ ) reported previously (Oya et al. 2014; Yen et al. 2017; Okoda et al. 2018), and their origins $\left(\alpha\right.$ and $\beta$ in Figure 5(a)) are offset by $1^{\prime \prime}-2^{\prime \prime}(\sim 150$ $-300 \mathrm{au}$ ) from the disk/envelope axis. This feature is also seen weakly in $\mathrm{C}^{18} \mathrm{O}$ emission over the same velocity range of $4.5-5.7 \mathrm{~km} \mathrm{~s}^{-1}$ (Figure 5(b)). 
Protostar
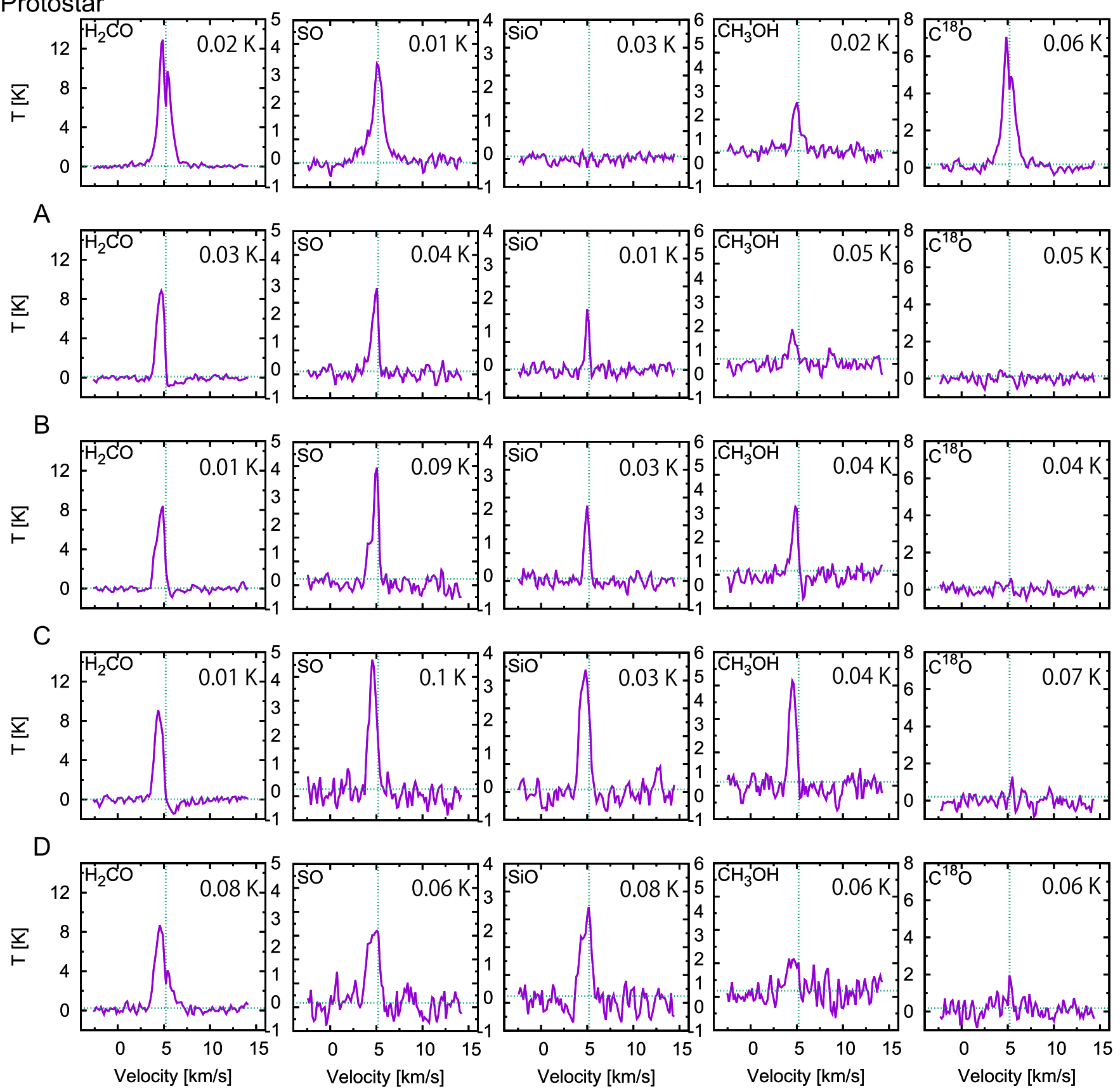

Figure 3. Molecular line profiles observed toward the four positions (A-D; see Figures 1 and 2) in the arc-like structure and at the protostellar position. These spectra are extracted from the apertures of 1 !" 0 . The horizontal green dashed lines represent each $3 \sigma$. The top right values indicate $\sigma$ for each line profile. The vertical green dashed lines represents the systemic velocity of the protostellar core, $5.2 \mathrm{~km} \mathrm{~s}^{-1}$ (Yen et al. 2017)

The SO emission also shows evidence of an extension to the northwest in the velocity maps of 5.1 and $5.5 \mathrm{~km} \mathrm{~s}^{-1}$ (Figure 4(b)). This emission is extended from the protostar along the same orientation as the linear structure (P.A. $140^{\circ}$ ) in the $5.1 \mathrm{~km} \mathrm{~s}^{-1}$ channel map (Figure 5). At $5.5 \mathrm{~km} \mathrm{~s}^{-1}$, additional emission appears further toward the northwest and shows a slight offset from the above axis. In contrast to the emission from $\mathrm{H}_{2} \mathrm{CO}, \mathrm{SO}$ is more structured and less continuous in the vicinity of the protostar. Nevertheless, the observed SO emission is consistent with the geometry seen in the $\mathrm{H}_{2} \mathrm{CO}$ emission. The morphology near the protostar in the $\mathrm{H}_{2} \mathrm{CO}$ and $\mathrm{C}^{18} \mathrm{O}$ emission and the apparent symmetry in the $\mathrm{SO}$ emission strongly suggest that these features are part of an outflow structure, rather than a structure formed from gas accreting toward the protostar as seen in the source Per-emb-2 by Pineda et al. (2020).

The possible secondary outflow is verified by the velocity structure. Figure 6(a) presents the position-velocity (PV) diagram for the $\mathrm{H}_{2} \mathrm{CO}$ emission along the northwest to southeast axis (P.A. $140^{\circ}$ ), centered at the protostellar position. The position axis is approximately along the linear feature seen in the $\mathrm{H}_{2} \mathrm{CO}$ emission (Figure 1(a)). The velocity toward the northwest remains roughly constant and only slightly blueshifted with respect to the systemic velocity. In contrast, toward 


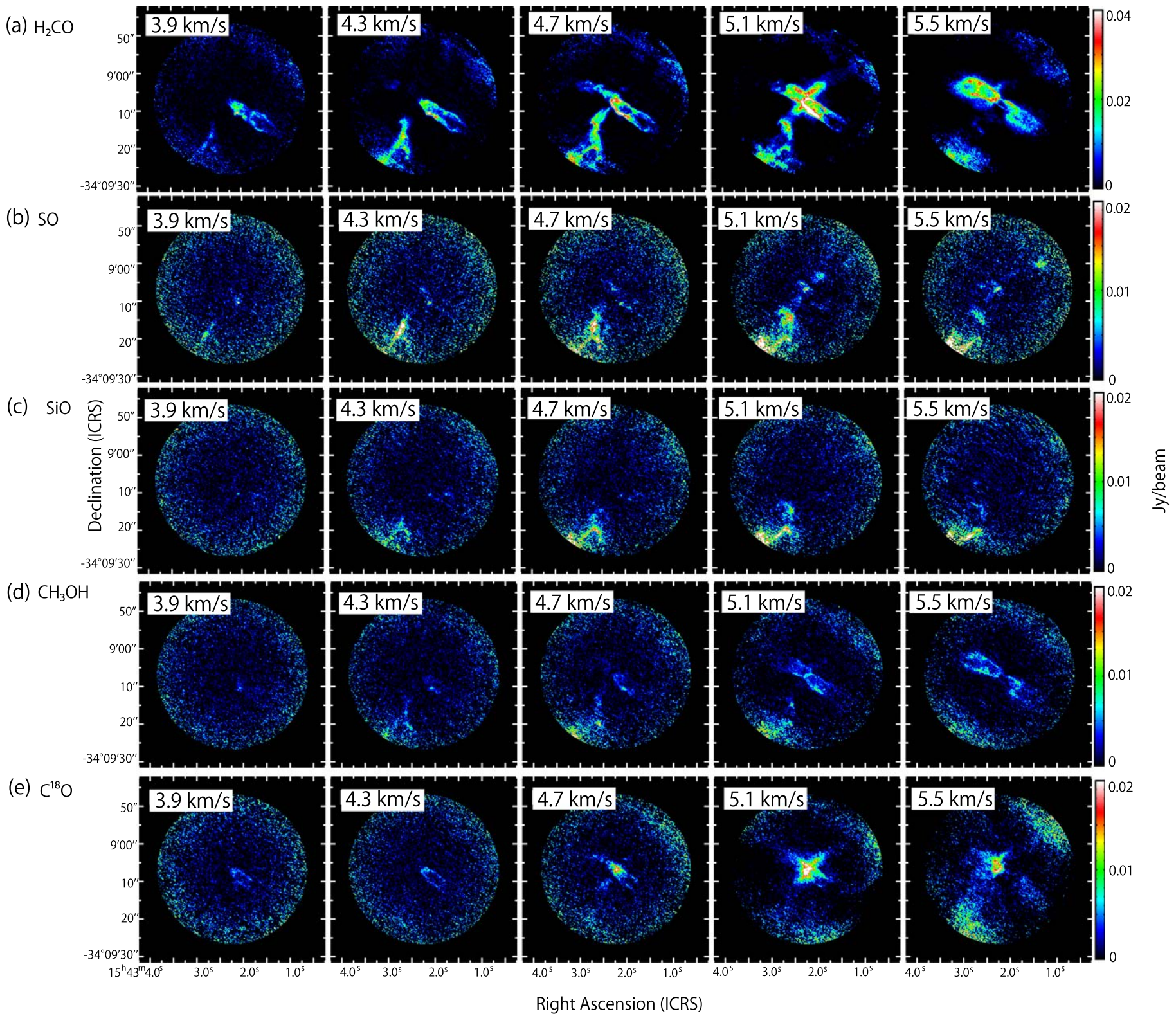

Figure 4. Velocity channel maps of $\mathrm{H}_{2} \mathrm{CO}$, $\mathrm{SO}, \mathrm{SiO}, \mathrm{CH}_{3} \mathrm{OH}$, and $\mathrm{C}^{18} \mathrm{O}$. Each panel represents the intensity averaged over a velocity range of $0.4 \mathrm{~km} \mathrm{~s}{ }^{-1}$ centered at the quoted velocity. For instance, the $3.9 \mathrm{~km} \mathrm{~s}^{-1}$ panel shows the average between 3.7 and $4.1 \mathrm{~km} \mathrm{~s}^{-1}$. The systemic velocity of the protostellar core is $5.2 \mathrm{~km} \mathrm{~s}$.

the southeast there is a clear velocity gradient, with the emission becoming more blueshifted with increasing distance from the protostar and toward the arc-like structure. Furthermore, as seen in the PV diagram, the velocity width increases up to about $1 \mathrm{~km} \mathrm{~s}^{-1}$ at the southeastern terminus (Position A; Figure 1(a)). This location is coincident with the northern tip of the arc-like structure. A similar velocity structure can be seen in the PV diagram for the $\mathrm{C}^{18} \mathrm{O}$ emission (Figure 6(b)). The velocity gradient is calculated to be about $1.2 \mathrm{~km} \mathrm{~s}^{-1}$ over $1200 \mathrm{au}$, assuming that the systemic velocity is $5.2 \mathrm{~km} \mathrm{~s}^{-1}$. This gradient is unexpectedly large if identified as infalling motion to the protostar. A more natural explanation is that the velocity gradient is associated with outflow motion. The lack of a redshifted component in the northwestern part is puzzling but may be due to less gas being affected by the outflow. Alternatively, this could be reproduced if accretion of material is not symmetric (Zhao et al. 2018). The slight shift of the points of origin of the linear structure near the protostar (Figure 5) can also be understood if these points of origin are part of this secondary outflow.

One may expect that the secondary outflow would be detected in the ${ }^{12} \mathrm{CO}$ lines, which are the most commonly observed outflow tracers. Indeed, a faint blueshifted component near the arc-like structure on the southeastern part can marginally be seen in the interferometric ${ }^{12} \mathrm{CO}(J=2-1)$ observations by Bjerkeli et al. (2016b) and Yen et al. (2017). This would be a remnant of part of the arc-like structure. On the other hand, the linear structure and its extension to the northwest are not seen in the ${ }^{12} \mathrm{CO}$ emission. The velocity shift is so close to that of the ambient gas that they are likely to be resolved out. The absence of the redshifted emission in the northwestern part is puzzling, as mentioned above. 
(a) $\mathrm{H}_{2} \mathrm{CO}$

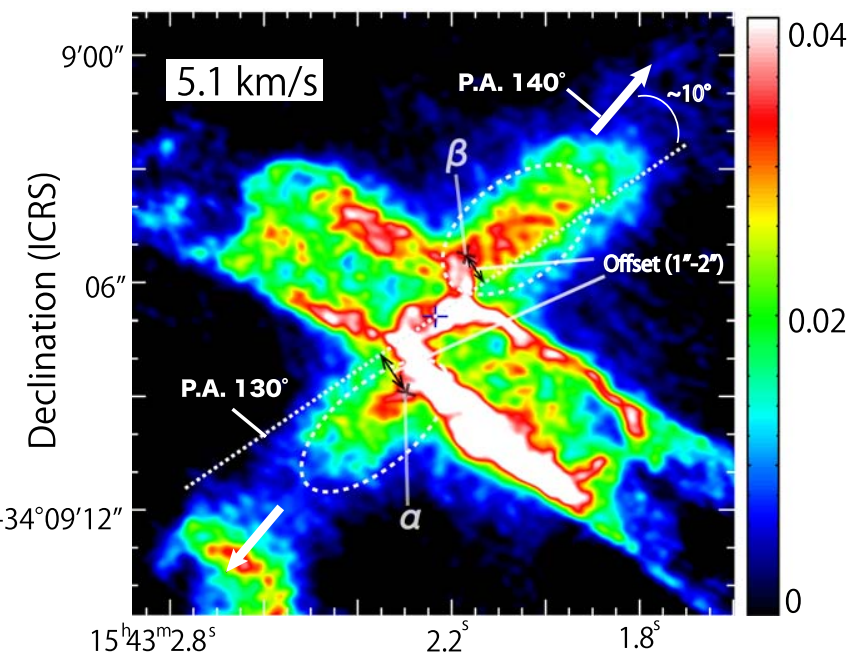

(b) $\mathrm{C}^{18} \mathrm{O}$

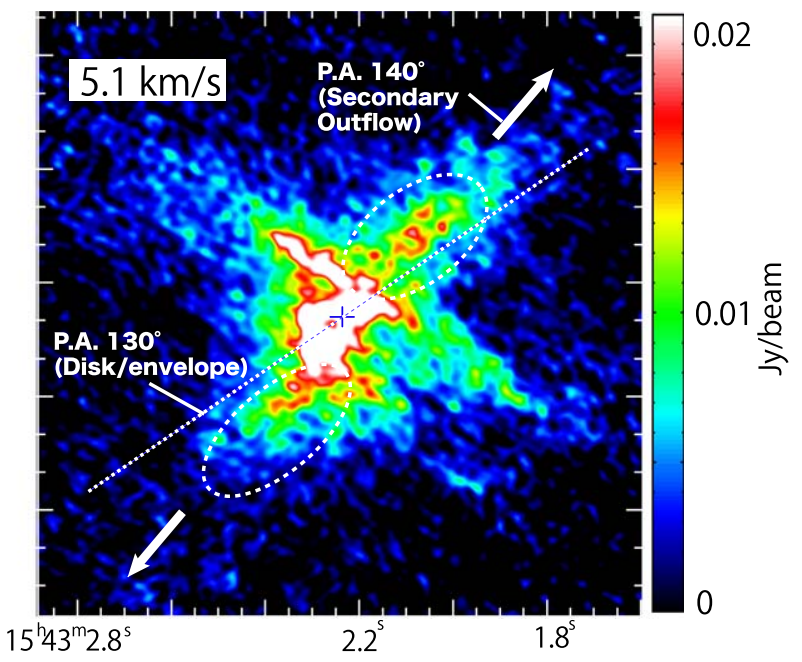

Right Ascension (ICRS)

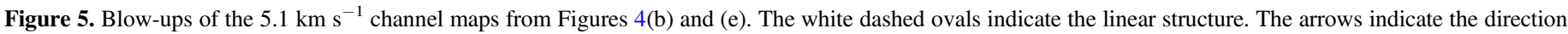

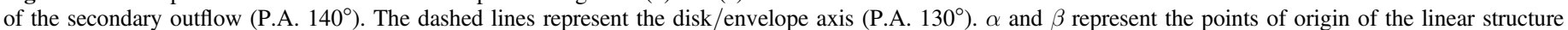

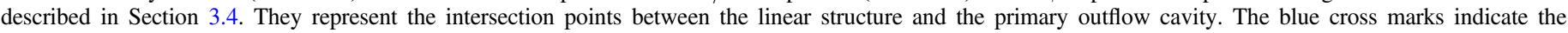
continuum peak position.
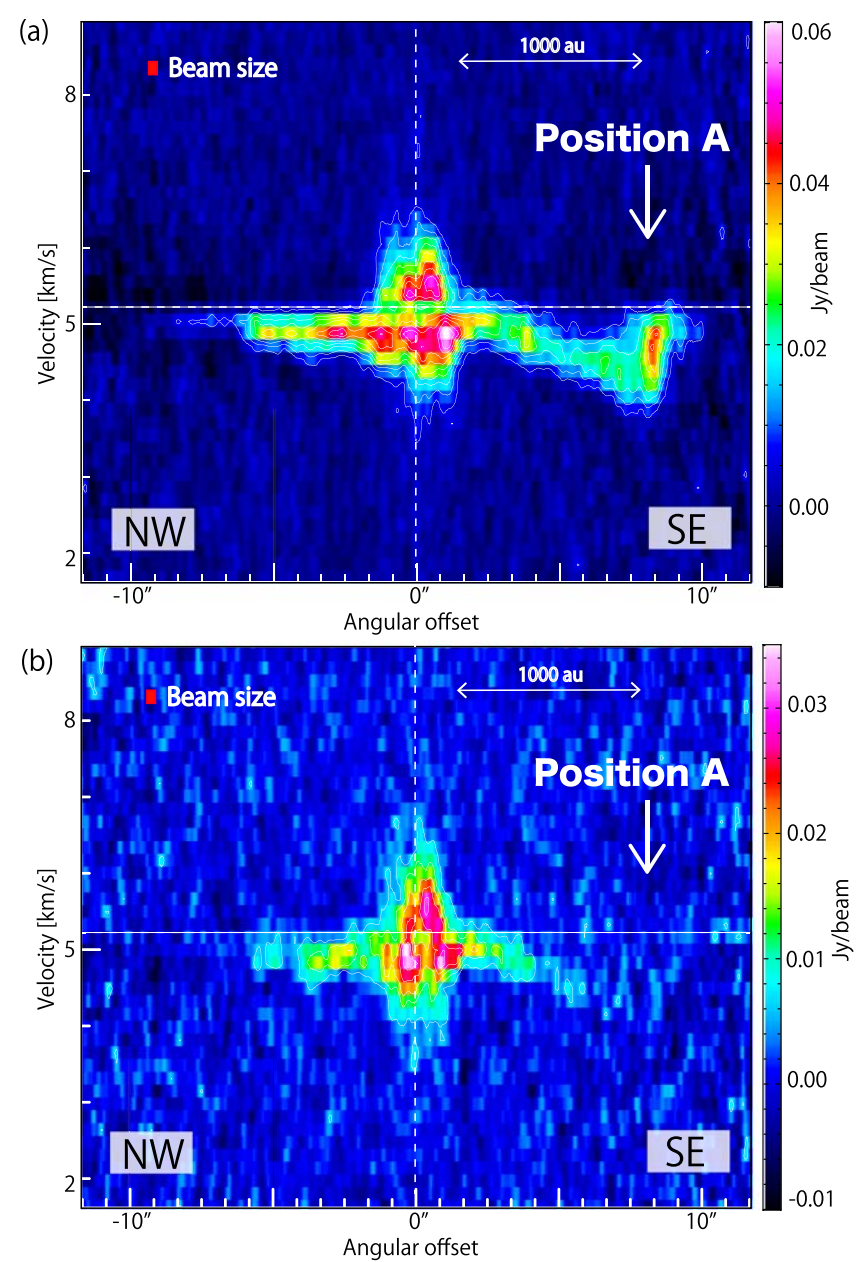

Figure 6. $\mathrm{PV}$ diagrams for (a) $\mathrm{H}_{2} \mathrm{CO}$ and (b) $\mathrm{C}^{18} \mathrm{O}$ along the northwest to southeast axis (P.A. $140^{\circ}$; Figure $1(\mathrm{a})$ ). The origin is the continuum peak position. The horizontal dashed line indicates the systemic velocity of the protostellar core $\left(5.2 \mathrm{~km} \mathrm{~s}^{-1}\right)$. The cut width is 1 !" 0 .

\section{Analyses of the Secondary Outflow}

\subsection{Dynamical Timescale of the Outflow and Outflow Mass}

We have identified a linear structure along the northwest to southeast axis (P.A. $140^{\circ}$ ) as well as an arc-like structure further to the southeast. A northwestern counterpart can marginally be seen in SO emission. This extended feature is most likely a secondary outflow, which has not been previously identified. Collimated features similar to the linear structure seen near the protostar are expected to form around young protostellar sources (e.g., Machida et al. 2008; Inutsuka 2012; Velusamy et al. 2014; Busch et al. 2020).

We evaluate the dynamical timescale of the secondary outflow from the $\mathrm{PV}$ diagram of the $\mathrm{H}_{2} \mathrm{CO}$ emission (Figure 6(a)). The distance in the plane of the sky and the shift in line-of-sight velocity from the systemic velocity are $1200 \mathrm{au}$ and $\sim 1.2 \mathrm{~km} \mathrm{~s}^{-1}$, respectively. Thus, the timescale is estimated to be $\sim 5000 \times \cot (i) \mathrm{yr}$, where $i$ is the inclination angle of the outflow axis ( $i=0^{\circ}$ for pole-on). Considering the morphology of the observed secondary outflow, the axis may be close to the plane of the sky $\left(i=90^{\circ}\right)$ and the dynamical timescale is regarded as an upper limit. The observed feature might also be a relic of a previous fast outflow, considering the relatively narrow observed line width (see Section 5.2). In this case, the observed velocities might be significantly lower than they were in the past, and the calculated dynamical timescale would be an overestimate. Note that the dynamical timescale of the primary outflow (P.A. $220^{\circ}$ ) is reported to be $10^{2}-10^{3}$ yr (Oya et al. 2014; Yıldız et al. 2015; Bjerkeli et al. 2016b). Hence, the secondary outflow would likely have formed before the primary outflow. The dynamical timescale of the secondary outflow is comparable to the time for depletion of $\mathrm{SiO}$ onto dust grains: $10^{3}-10^{4} \mathrm{yr}$ if the $\mathrm{H}_{2}$ density is roughly $10^{5}-10^{6} \mathrm{~cm}^{-3}$ (e.g., Caselli et al. 1999). Thus, if $\mathrm{SiO}$ molecules were liberated from dust grains in the past by shocks related to a secondary outflow, they would be able to survive in the gas phase until the present day. We roughly 
Table 3

Column Densities $\left[10^{14} \mathrm{~cm}^{-2}\right]^{\mathrm{a}}$

\begin{tabular}{lccccc}
\hline \hline Molecule & Protostar $^{\mathrm{b}}$ & $\mathrm{A}^{\mathrm{c}}$ & $\mathrm{B}^{\mathrm{c}}$ & $\mathrm{C}^{\mathrm{c}}$ & $\mathrm{D}^{\mathrm{c}}$ \\
\hline $\mathrm{H}_{2} \mathrm{CO}$ & $0.4-2$ & $0.2-2$ & $0.2-2$ & $0.2-3$ & $0.2-3$ \\
$\mathrm{SO}$ & $0.4-1$ & $0.2-2$ & $0.3-3$ & $0.5-5$ & $0.4-3$ \\
$\mathrm{SiO}$ & $<0.03^{\mathrm{d}}$ & $0.01-0.8$ & $0.02-1$ & $0.07-5$ & $0.05-3$ \\
$\mathrm{CH}_{3} \mathrm{OH}$ & $0.7-6$ & $1-6$ & $2-9$ & $3-20$ & $2-10$ \\
$\mathrm{C}^{18} \mathrm{O}$ & $55-64$ & $<1^{\mathrm{d}}$ & $<0.8^{\mathrm{d}}$ & $<2^{\mathrm{d}}$ & $<2^{\mathrm{d}}$ \\
\hline
\end{tabular}

Notes.

a The positions are shown in Figures 1 and 2 .

b The temperature and the $\mathrm{H}_{2}$ density are assumed to be $54 \mathrm{~K}$ (Okoda et al. 2020) and $10^{5}-10^{7} \mathrm{~cm}^{-3}$, respectively. A range of column density is shown for each molecule.

c The temperature and the $\mathrm{H}_{2}$ density are assumed to be $20-80 \mathrm{~K}$ and $10^{5}-10^{6} \mathrm{~cm}^{-3}$, respectively. A range of column density is shown for each molecule.

${ }^{\mathrm{d}}$ The $3 \sigma$ upper limits, where $\sigma$ is shown in Figure 3.

estimate the total mass within the observed secondary structure seen in SO emission to be $10^{-5}-10^{-4} M_{\odot}$, where we use the apparent size and an assumed $\mathrm{H}_{2}$ density of $10^{5}-10^{6} \mathrm{~cm}^{-3}$ based on the critical densities of the observed lines. Similarly, we estimate the mass of the primary outflow from the $\mathrm{H}_{2} \mathrm{CO}$ emission to be $10^{-4}-10^{-3} M_{\odot}$, assuming the same range of $\mathrm{H}_{2}$ density.

\subsection{Molecular Abundances around the Arc-like Structure}

In order to compare the molecular abundances of the IRAS 15398-3359 with other outflow shock regions, we derive the lower limits to the abundance ratio for several molecules relative to $\mathrm{C}^{18} \mathrm{O}$ at the four positions $(\mathrm{A}-\mathrm{D})$ and the abundance ratio at the protostar position (Figures 1 and 2). Positions A and $\mathrm{D}$ are the bending points from the linear structure toward the arc-like structure and the south of the arc-like structure, respectively, while positions $\mathrm{B}$ and $\mathrm{C}$ are the local peak positions of $\mathrm{CH}_{3} \mathrm{OH}$ in Figure 2(b). We calculate the column densities of $\mathrm{H}_{2} \mathrm{CO}, \mathrm{SO}, \mathrm{SiO}$, and $\mathrm{CH}_{3} \mathrm{OH}$ as well as the upper limit of $\mathrm{C}^{18} \mathrm{O}$ by using the non-LTE radiative transfer code, RADEX $^{42}$ (van der Tak et al. 2007), toward these positions, assuming that the gas temperature is $20-80 \mathrm{~K}$ and the $\mathrm{H}_{2}$ density is $10^{5}-10^{6} \mathrm{~cm}^{-3}$ (Table 3 ). Regarding the $\mathrm{C}^{18} \mathrm{O}$ column density at the four positions, we use the upper limits to the intensity and the mean line width of other molecular lines $\left(\mathrm{H}_{2} \mathrm{CO}, \mathrm{SO}, \mathrm{SiO}\right.$, and $\left.\mathrm{CH}_{3} \mathrm{OH}\right)$. Any extended component traced by the $\mathrm{C}^{18} \mathrm{O}$ emission at the systemic velocity may be resolved out. In this case, the upper limits to the $\mathrm{C}^{18} \mathrm{O}$ intensity might be underestimated. However, we here discuss the molecular abundances in the arc-like structure, which is blueshifted from the systemic velocity by $0.5 \mathrm{~km} \mathrm{~s}^{-1}$. Such a compact structure should be observed even in the $\mathrm{C}^{18} \mathrm{O}$ emission without the resolved-out problem. Moreover, the maximum recoverable size (12!.8) is larger than the arc-like structure (at most $10^{\prime \prime}$ ). For these reasons, the resolved-out effect should not affect the molecular abundances significantly. For the protostar position, the $\mathrm{C}^{18} \mathrm{O}$ column density is derived to be $(5.5-6.4) \times 10^{15} \mathrm{~cm}^{-2}$, where the gas temperature and

\footnotetext{
${ }^{42}$ The collisional rates of $\mathrm{H}_{2} \mathrm{CO}, \mathrm{SO}, \mathrm{SiO}, \mathrm{CH}_{3} \mathrm{OH}$, and $\mathrm{C}^{18} \mathrm{O}$ are originally calculated by Wiesenfeld \& Faure (2013), Lique et al. (2006), Balanća et al. (2018), Rabli \& Flower (2010), and Yang et al. (2010), respectively.
}

the $\mathrm{H}_{2}$ density are assumed to be $54 \mathrm{~K}$ (Okoda et al. 2020) and $10^{5}-10^{7} \mathrm{~cm}^{-3}$, respectively. Then, the abundance ratios relative to $\mathrm{C}^{18} \mathrm{O}$ are evaluated from the column densities, where the same temperature is assumed for the molecular species and $\mathrm{C}^{18} \mathrm{O}$.

Only lower limits are obtained for the abundance ratios at all the four positions. Nevertheless, the abundances of $\mathrm{SO}, \mathrm{SiO}$, and $\mathrm{CH}_{3} \mathrm{OH}$ are found to be significantly enhanced in the shocked region in comparison with the protostar position (Table 4). Moreover, the abundance ratios are as high as those reported for L1157-B1 (Table 4), further supporting the outflow shock. We note, however, that the physical conditions of L1157-B1 are likely different from those of IRAS 15398 -3359. L1157-B1 shows a broad line width of SiO. Hence, we also compare the $\mathrm{SiO}$ abundance of the arc-like structure to that of the shocked region with the narrow $\mathrm{SiO}$ line in the Class I protostellar source, HH7-11 (SVS13-A) (Codella et al. 1999; $t_{\text {des }} \sim 10^{4} \mathrm{yr}$ ). We estimate the $\mathrm{SiO}$ abundance relative to $\mathrm{H}_{2}$ by using the nominal $\mathrm{C}^{18} \mathrm{O}$ fractional abundance of $1.7 \times 10^{-7}$ (Frerking et al. 1982). In Table 4, the abundance of the arc-like structure is much higher than that found for HH7-11 (SVS13A). This result seems to be related to the shorter timescale of the secondary outflow than that of the HH7-11 (SVS13-A) outflow, $\sim 10^{5} \mathrm{yr}$ (Lefloch et al. 1998).

\section{Origin of the Secondary Outflow}

\subsection{Scenario 1: Two Outflows Driven by a Binary System}

It is well known that some Class 0 protostellar sources contain a binary or multiple system (e.g., Tobin et al. 2016b, 2018). A possible explanation for the secondary outflow is that IRAS $15398-3359$ is a binary system, launching outflows in two different directions with respect to the plane of the sky. Such binary/multiple systems launching more than one distinct outflow in different directions have been previously observed within IRAS 16293-2422 Source A (van der Wiel et al. 2019; Maureira et al. 2020), BHR71 (Zapata et al. 2018; Tobin et al. 2019), NGC 1333 IRAS2A (Tobin et al. 2015), and NGC 2264 CMM3 (Watanabe et al. 2017). In all these cases, however, the binaries are separated by more than 40-50 au. Most binary sources observed to have a circumbinary disk/envelope structure show only a single outflow or parallel outflows, e.g., BHB07-11 (Alves et al. 2017), L1448 IRS3B-a, b (Lee et al. 2015; Tobin et al. 2016a), and L1551NE (Reipurth et al. 2000; Lim et al. 2016). An exception is the case of VLA 1623A. Hara et al. (2020) report that this source is a close binary system with a separation of $34 \mathrm{au}$, and two molecular outflows are inclined by $70^{\circ}$ to each other across the plane of the sky.

A high-resolution observation $(\sim 30 \mathrm{au})$ of IRAS 15398 -3359 with ALMA by Okoda et al. (2018) shows a single peak in the dust continuum, indicating that this source is not a wide binary. In addition, they find a well defined Keplerian disk structure with a size of 40 au in the SO line. If IRAS 15398 -3359 is a close binary system instead of being two independent systems that happen to be accidentally aligned in the line of sight of our observations, it poses the very difficult question of how to create such a complex system from a molecular cloud core. In principle, a single molecular cloud core can have a very complicated distribution of internal angular momentum, but there is no theory to explain the formation of binary stars with an apparent separation much 
Table 4

Relative Molecular Abundance Ratios with Respect to the $\mathrm{C}^{18} \mathrm{O}$ Emission

\begin{tabular}{|c|c|c|c|c|c|c|c|}
\hline Molecule & Protostar $^{\mathrm{a}}$ & $\mathrm{A}^{\mathrm{a}}$ & $\mathrm{B}^{\mathrm{a}}$ & $\mathrm{C}^{\mathrm{a}}$ & $\mathrm{D}^{\mathrm{a}}$ & $\mathrm{L} 1157-\mathrm{B} 1^{\mathrm{b}}$ & HH7-11 (SVS13-A) \\
\hline$\left[\mathrm{H}_{2} \mathrm{CO}\right] /\left[\mathrm{C}^{18} \mathrm{O}\right]$ & $0.005-0.03$ & $>0.2$ & $>0.2$ & $>0.1$ & $>0.1$ & $0.5-1$ & $\cdots$ \\
\hline$[\mathrm{SO}] /\left[\mathrm{C}^{18} \mathrm{O}\right]$ & $0.007-0.02$ & $>0.2$ & $>0.4$ & $>0.3$ & $>0.2$ & $0.5-0.8$ & $\cdots$ \\
\hline$[\mathrm{SiO}] /\left[\mathrm{C}^{18} \mathrm{O}\right]$ & $<0.001$ & $>0.01$ & $>0.03$ & $>0.04$ & $>0.02$ & 0.1 & $\ldots$ \\
\hline$\left[\mathrm{CH}_{3} \mathrm{OH}\right] /\left[\mathrm{C}^{18} \mathrm{O}\right]$ & $0.01-0.1$ & $>1$ & $>2$ & $>2$ & $>1$ & $0.8-4$ & $\ldots$ \\
\hline$X(\mathrm{SiO})^{\mathrm{c}}\left[10^{-9}\right]$ & & 1.7 & 5.1 & 6.8 & 3.4 & 17 & $0.08-0.3^{\mathrm{d}}$ \\
\hline
\end{tabular}

Notes.

a These values are derived by assuming the same temperature for the two molecular species for the ratio.

b The abundance ratios in the outflow-shocked region of L1157-B1. Values are extracted from Bachiller \& Pérez Gutiérrez (1997).

${ }^{c} X(\mathrm{SiO})$ indicates the $\mathrm{SiO}$ abundance relative to $\mathrm{H}_{2}$ estimated by using the normal $\mathrm{C}^{18} \mathrm{O}$ abundance, $1.7 \times 10^{-7}($ Frerking et al. 1982$)$.

${ }^{\mathrm{d}}$ These values are reported in Lefloch et al. (1998), Codella et al. (1999).

smaller than the above ALMA resolution ( $\sim 30 \mathrm{au})$ and with very different rotation axes. Indeed, MHD simulations by Matsumoto et al. (2017) reveal that a single outflow is launched in the case of a complex angular momentum distribution in the core. Note also that it is very unlikely that two, almost orthogonal, outflows would be launched from the circumbinary disk. We thus conclude that the binary hypothesis for the secondary outflow is unlikely, although we cannot completely rule out the possibility of a tight binary $(<30 \mathrm{au})$ system or that of a binary system whose components are aligned close to the line of sight. More observations at higher resolution will be required to investigate the multiplicity of IRAS 15398-3359.

\subsection{Scenario 2: Outflow Outburst and Reorientation}

A second possible explanation for the observed secondary structure is a past reorientation of the outflow launched from a single protostar. Assuming that the likelihood of outflow reorientation is random, the observed orthogonality of the secondary and primary outflows suggests a low expectation for this specific geometry, especially if the secondary outflow lies nearly in the plane of the sky as in the case of the primary outflow. This difficulty can be mitigated if the secondary outflow is a relic and the fast-moving component expected along the outflow has already been dissipated. In this case, the observed outflow direction may be significantly inclined with respect to the plane of the sky and the observed velocity may be close to the true velocity of the present relic.

The dissipation timescale of the high-velocity component can be roughly calculated as (Codella et al. 1999)

$$
t_{\mathrm{dis}}=\frac{8}{3} \frac{\rho}{\rho_{1}} \frac{r}{v}
$$

where $\rho / \rho_{1}$ is the ratio of the mass density of the shocked gas to that of the ambient gas, $r$ the size of the shocked region, and $v$ the shock velocity. In the case of IRAS 15398-3359, we assume the shock velocity to be $10 \mathrm{~km} \mathrm{~s}^{-1}$ and employ the size of the shocked region of 300 au according to a typical width of the arc-like structure (Figure 1). In addition, the ratio $\rho / \rho_{1}$ is assumed to be 10 . The turbulent dissipation timescale is estimated to be $\sim 4000 \mathrm{yr}$ based on the above assumptions. This timescale is comparable to the dynamical timescale of the secondary outflow $(\sim 5000 \times \cot (i)$ yr $)$. Thus, the absence of the high-velocity component seems reasonable.

It is further worth pointing out two specific features of IRAS 15398-3359 in relation to the reorientation hypothesis. First, the protostellar mass is reported to be $0.007 M_{\odot}$ on the basis of the disk Keplerian motion (Okoda et al. 2018). This mass is lower than the expected mass of the first hydrostatic core proposed by star formation theories $\left(0.01-0.05 M_{\odot}\right.$; Larson 1969; Masunaga et al. 1998; Saigo \& Tomisaka 2006; Inutsuka 2012). Thus, IRAS 15398-3359 appears to be in the earliest stages of protostellar evolution, a notion reinforced by the significantly larger reported envelope mass, $0.5-1.2 M_{\odot}$ (Kristensen et al. 2012; Jørgensen et al. 2013). Second, past episodic accretion events have been suggested for this source by Jørgensen et al. (2013) and Bjerkeli et al. (2016a). Jørgensen et al. (2013) find a ring structure of $\mathrm{H}^{13} \mathrm{CO}^{+}$at a scale of $150-200$ au around the protostar. The lack of $\mathrm{H}^{13} \mathrm{CO}^{+}$interior to the ring is inconsistent with the present heating rate from the central protostar; however, a previous burst of enhanced luminosity due to an accretion burst would have removed that $\mathrm{H}^{13} \mathrm{CO}^{+}$through chemical reaction with sublimated $\mathrm{H}_{2} \mathrm{O}$ from dust grains. The authors predict that the accretion burst occurred $10^{2}-10^{3} \mathrm{yr}$ ago, consistent with the dynamical timescale of the primary outflow (Oya et al. 2014; Bjerkeli et al. 2016b). Bjerkeli et al. (2016a) report that the HDO $\left(1_{0,1}-0_{0,0}\right)$ emission is localized on the cavity wall in the vicinity of the protostar, interpreting that this is also due to a past accretion burst. The timescale for the outflow reorientation would be similar to the interval of episodic accretion in the case when the angular momentum of the gas in a molecular core is nonuniform (see below).

Assuming that the secondary structure reflects a previous change in the outflow direction for IRAS 15398-3359, can the observed velocity and orientation of the feature be reconciled? Relatively high-velocity shocks $\left(>5-20 \mathrm{~km} \mathrm{~s}^{-1}\right)$ are required to liberate $\mathrm{SiO}$ from dust grains (e.g., Caselli et al. 1997; Jiménez-Serra et al. 2008). Thus, the low velocity shift of $\sim 1 \mathrm{~km} \mathrm{~s}^{-1}$ of the $\mathrm{SiO}$ arc-like structure needs to be considered carefully.

As discussed above, the possibility that the secondary outflow velocity is underestimated due to the structure lying very close to the plane of the sky is small. Alternatively, a more likely hypothesis is that the observed secondary outflow represents a relic structure that is no longer powered by current mass ejection from the protostar and inner disk. Thus, the fast component of the secondary outflow should have dissipated through interaction with ambient gas, leaving only the observed motions close to the systemic velocity. This is also the reason why we can see the linear structure from the vicinity of the protostar. Previous observations of low-velocity features in $\mathrm{SiO}$ have also suggested this hypothesis (Lefloch et al. 1998; 
Codella et al. 1999; López-Sepulcre et al. 2016). The relatively narrow line widths observed for the shock tracer lines described in Section 3.2 are consistent with this picture.

We must also reconcile the orientation of the secondary outflow, which is almost perpendicular to the angular momentum axis of the currently accreting gas. If the angular momentum of the episodically accreting gas varies with time, the direction of the outflow axis may change drastically, even as much as observed in this source. According to Misugi et al. (2019), the angular momentum of the gas in a molecular core is related to the degree of centroid velocity fluctuations within the parental filamentary molecular cloud, resulting in the relation between angular momentum and cloud mass determined by the Kolmogorov power spectrum of weak (i.e., subsonic or transonic) turbulence. In this analysis, the total angular momentum of a star-forming core is a vector sum of the various angular momenta of turbulent fluid elements whose directions are almost randomly oriented. The summation over angular momentum vectors inevitably includes cancellation of opposite components of angular momentum vectors.

The identification of such an origin for the core rotation has important implications, because the actual collapse process of the core is not homologous but rather undergoes a "runaway" process where a central dense region collapses first and the outer regions accrete onto the central region later. Thus, episodic accretion events with very different angular momenta are expected to occur during this runaway collapse process. Applying this model to the early evolution of the IRAS 15398 -3359 protostellar core, and recognizing that the mass of the central protostar is extremely low, there should only have been a very few discrete episodic accretion events and random reorientations of angular momentum. Moreover, Okoda et al. (2018) report that the central velocity of the disk structure observed with the SO line is $5.5 \mathrm{~km} \mathrm{~s}^{-1}$, which is shifted 0.3 $\mathrm{km} \mathrm{s}^{-1}$ from the systemic velocity of the protostellar core at $5.2 \mathrm{~km} \mathrm{~s}^{-1}$ (Yen et al. 2017). This previous result may also be naturally caused by the continual accretion of fluid elements that have various momenta as well as various angular momenta. The observed secondary outflow feature provides plausible observational evidence for this picture. It is interesting to note that a change in the angular momentum axis of the accreting gas has also recently been suggested observationally for some other young protostellar sources (Sakai et al. 2019; Zhang et al. 2019; Gaudel et al. 2020).

If the rotation axis is misaligned with the global magnetic field direction, the MHD simulations show that the directions of the jet/outflow and disk change randomly over time (Matsumoto \& Tomisaka 2004; Hirano et al. 2020; Machida et al. 2020). Since the misalignment can naturally be caused by the above picture, this mechanism would also contribute to the outflow reorientation of this source. Note that the global direction of the magnetic field around IRAS 15398-3359 is almost in the primary outflow direction (Redaelli et al. 2019).

\subsection{Future Directions}

Unfortunately, the above interesting theoretical process is not yet well studied theoretically because most of the numerical MHD simulations for the formation of protostars and disks have been performed with the simplest initial condition for the rotation, i.e., rigid-body rotation throughout the core (e.g., Inutsuka 2012; Tsukamoto 2016; Wurster \& Li 2018; Machida $\&$ Basu 2019). Such turbulent motion, however, could be important for variation of angular momentum within the core as well as the thermal pressure of the gas.

We propose the reorientation scenario as one possibility to account for this observation. To understand the interesting feature of the secondary outflow, we will need additional observations to investigate other possibilities carefully, particularly the existence of the close binary system $(<30 \mathrm{au})$. For testing the close binary hypothesis, centimeter-wave observations at a high angular resolution $(<30 \mathrm{au})$ are essential to resolve the components without obscuration from the optically thick dust continuum. Mosaic observations with ACA would be useful to explore the environment around this source in detail. It is also interesting to observe the velocity field of the parent core at a high angular resolution in order to investigate the distribution of the angular momentum as previously done at a larger scale for other sources (e.g., Caselli et al. 2002). Furthermore, observations in other molecular lines $(\mathrm{CCH}, \mathrm{CS}$, c- $\mathrm{C}_{3} \mathrm{H}_{2}, \mathrm{~N}_{2} \mathrm{H}^{+}$, and $\mathrm{H}^{13} \mathrm{CO}^{+}$etc.) would promote our understanding of the newly found structures, and these are now in progress in the FAUST program.

\section{Conclusions}

We have uncovered an interesting feature in a direction almost perpendicular to the primary outflow in IRAS 15398 -3359 . This is most likely the relic of a secondary outflow ejected from the single protostar at the center of this system. The observational results of this paper present an important implication for the earliest stage of star formation. The main results are listed below.

1. We have identified an arc-like structure in $\mathrm{SO}, \mathrm{SiO}$, and $\mathrm{CH}_{3} \mathrm{OH}$ line emission. Since these molecular species are known to be shock tracers, the arc-like structure is most likely a shock region produced by a relic outflow. The molecular abundances of $\mathrm{H}_{2} \mathrm{CO}, \mathrm{SO}, \mathrm{SiO}$, and $\mathrm{CH}_{3} \mathrm{OH}$ in the arc-like structure are clearly enhanced in comparison with those at the protostar position, and they are consistent with those reported for shocked regions in the source L1157-B1.

2. The $\mathrm{H}_{2} \mathrm{CO}$, SO, and $\mathrm{C}^{18} \mathrm{O}$ line emission produces a linear feature around the protostar. The $\mathrm{SO}$ emission also reveals an additional structure to the northwest. In the PV diagram of the $\mathrm{H}_{2} \mathrm{CO}$ and $\mathrm{C}^{18} \mathrm{O}$ emission, the velocity increases with increasing distance from the protostar toward the southeast. Such morphological and kinematic features are consistent with those expected from an outflow.

3 . We roughly estimate the dynamical timescale of the secondary outflow to be $\sim 5000 \times \cot (i)$ yr $\left(i=0^{\circ}\right.$ for poleon). The timescale is similar to that of depletion of $\mathrm{SiO}$ onto dust grains, which is $10^{3}-10^{4} \mathrm{yr}$.

4. As previously reported by Okoda et al. (2018), the Keplerian disk structure around IRAS 15398-3359 is seen in the SO line emission at a resolution of $30 \mathrm{au}$, and the dust continuum maps show only a single peak. Given this geometry, the launch of two almost orthogonal outflows from the circumbinary disk $(<30 \mathrm{au})$ is very unlikely, although we cannot completely exclude a tight binary hypothesis. Observations at higher resolution are needed to examine the possibility further.

5. We thus hypothesize that the secondary outflow is a relic of a past reorientation of the outflow launched from a single protostar. The narrow line width of the $\mathrm{SiO}$ emission implies that the arc-like structure has dissipated the turbulent motions associated with the earlier shocks. The change in the direction 
of the outflow axis may be related to a nonuniform distribution of internal angular momentum in the molecular core, advected onto the central region via episodic accretion. Such events may occur during the earliest stages of protostar formation like IRAS 15398-3359.

The authors thank the anonymous reviewer for invaluable comments. They are also grateful to Hauyu Baobab Liu for useful discussions. This paper makes use of the following ALMA data set: ADS/JAO.ALMA\# 2018.1.01205.L (PI: Satoshi Yamamoto). ALMA is a partnership of the ESO (representing its member states), the NSF (USA) and NINS (Japan), together with the NRC (Canada) and the NSC and ASIAA (Taiwan), in cooperation with the Republic of Chile. The Joint ALMA Observatory is operated by the ESO, the AUI/NRAO, and the NAOJ. The authors thank to the ALMA staff for their excellent support. This work is supported by the projects PRIN-INAF-MAIN-STREAM 2017 "Protoplanetary disks seen through the eyes of new-generation instruments", "ARIEL and the astrochemical link between circumstellar disks and planets, and the European Research Council (ERC) under the European Union's Horizon 2020 research and innovation programmes: "The Dawn of Organic Chemistry" (DOC), grant agreement No. 741002, and "Astro-Chemistry Origins" (ACO), grant No. 811312. The National Radio Astronomy Observatory is a facility of the National Science Foundation operated under cooperative agreement by Associated Universities, Inc. I.J.-S. has received partial support from the Spanish FEDER (project number ESP2017-86582-C4-1-R) and the State Research Agency (AEI; project number PID2019-105552RB-C41). D.J. is supported by NRC Canada and by an NSERC Discovery Grant. This project is also supported by a Grant-in-Aid from Japan Society for the Promotion of Science (KAKENHI: Nos. $18 \mathrm{H} 05222$, 19H05069, 19K14753). Yuki Okoda thanks the Advanced Leading Graduate Course for Photon Science (ALPS) and Japan Society for the Promotion of Science (JSPS) for financial support.

\section{ORCID iDs}

Yuki Okoda (ib https://orcid.org/0000-0003-3655-5270 Yoko Oya (iD https://orcid.org/0000-0002-0197-8751 Logan Francis (iD https://orcid.org/0000-0001-8822-6327 Doug Johnstone (iD https://orcid.org/0000-0002-6773-459X Shu-ichiro Inutsuka (i) https://orcid.org/0000-0003-4366-6518 Cecilia Ceccarelli ii https://orcid.org/0000-0001-9664-6292 Claudio Codella (iD https://orcid.org/0000-0003-1514-3074 Claire Chandler (iD https://orcid.org/0000-0002-7570-5596 Nami Sakai (i) https://orcid.org/0000-0002-3297-4497 Yuri Aikawa (i) https://orcid.org/0000-0003-3283-6884 Felipe O. Alves (i) https://orcid.org/0000-0002-7945-064X Nadia Balucani (iD https://orcid.org/0000-0001-5121-5683 Eleonora Bianchi (i) https://orcid.org/0000-0001-9249-7082 Mathilde Bouvier (iD https://orcid.org/0000-0003-0167-0746 Paola Caselli (iD https://orcid.org/0000-0003-1481-7911 Marta De Simone (i) https://orcid.org/0000-0001-5659-0140 Siyi Feng (D) https://orcid.org/0000-0002-4707-8409 Francesco Fontani (ib https://orcid.org/0000-0003-0348-3418 Tetsuya Hama (iD https://orcid.org/0000-0002-4991-4044 Tomoyuki Hanawa (1) https://orcid.org/0000-0002-7538-581X Eric Herbst (iD https://orcid.org/0000-0002-4649-2536 Tomoya Hirota (iD https://orcid.org/0000-0003-1659-095X Muneaki Imai (ib https://orcid.org/0000-0002-5342-6262
Andrea Isella (ib https://orcid.org/0000-0001-8061-2207 Izaskun Jímenez-Serra (iD https://orcid.org/0000-00034493-8714

Claudine Kahane (ib) https://orcid.org/0000-0003-1691-4686 Laurent Loinard (ib https://orcid.org/0000-0002-5635-3345 Ana López-Sepulcre (iD https://orcid.org/0000-00026729-3640

Luke T. Maud (1) https://orcid.org/0000-0002-7675-3565 María José Maureira (i) https://orcid.org/0000-00027026-8163

Francois Menard (iD https://orcid.org/0000-0002-1637-7393 Anna Miotello (i) https://orcid.org/0000-0002-7997-2528 George Moellenbrock (i) https://orcid.org/0000-00023296-8134

Shoji Mori (i) https://orcid.org/0000-0002-7002-939X

Riouhei Nakatani (i) https://orcid.org/0000-0002-1803-0203

Hideko Nomura (10) https://orcid.org/0000-0002-7058-7682

Yasuhiro Oba (i) https://orcid.org/0000-0002-6852-3604

Satoshi Ohashi (i) https://orcid.org/0000-0002-9661-7958

Jaime E. Pineda (1) https://orcid.org/0000-0002-3972-1978

Albert Rimola (10) https://orcid.org/0000-0002-9637-4554

Takeshi Sakai (10 https://orcid.org/0000-0003-4521-7492

Dominique Segura-Cox (i) https://orcid.org/0000-00033172-6763

Brian Svoboda (iD https://orcid.org/0000-0002-8502-6431 Vianney Taquet (i) https://orcid.org/0000-0003-0407-7489 Leonardo Testi (ib https://orcid.org/0000-0003-1859-3070 Serena Viti (1D https://orcid.org/0000-0001-8504-8844 Naoki Watanabe (i) https://orcid.org/0000-0001-8408-2872 Yoshimasa Watanabe (iD https://orcid.org/0000-00029668-3592

Ci Xue (i) https://orcid.org/0000-0003-2760-2119

Yichen Zhang (i) https://orcid.org/0000-0001-7511-0034

\section{References}

Alves, F. O., Girart, J. M., Caselli, P., et al. 2017, A\&A, 603, L3 Arce, H. G., Mardones, D., Corder, S. A., et al. 2013, ApJ, 774, 39 Bachiller, R., \& Pérez Gutiérrez, M. 1997, ApJL, 487, L93

Balança, C., Dayou, F., Faure, A., et al. 2018, MNRAS, 479, 2692 Bjerkeli, P., Jørgensen, J. K., Bergin, E. A., et al. 2016a, A\&A, 595, A39 Bjerkeli, P., Jørgensen, J. K., \& Brinch, C. 2016b, A\&A, 587, A145 Busch, L. A., Belloche, A., Cabrit, S., et al. 2020, A\&A, 633, A126 Caselli, P., Benson, P. J., Myers, P. C., et al. 2002, ApJ, 572, 238 Caselli, P., Hartquist, T. W., \& Havnes, O. 1997, A\&A, 322, 296 Caselli, P., Walmsley, C. M., Tafalla, M., et al. 1999, ApJL, 523, L165 Codella, C., Bachiller, R., \& Reipurth, B. 1999, A\&A, 343, 585 Codella, C., Ceccarelli, C., Bianchi, E., et al. 2020, A\&A, 635, A17 Codella, C., Lefloch, B., Ceccarelli, C., et al. 2010, A\&A, 518, L112 Dzib, S. A., Loinard, L., Ortiz-León, G. N., et al. 2018, ApJ, 867, 151 Endres, C. P., Schlemmer, S., Schilke, P., et al. 2016, JMoSp, 327, 95 Favre, C., Vastel, C., Jimenez-Serra, I., et al. 2020, A\&A, 635, A189 Feng, S., Codella, C., Ceccarelli, C., et al. 2020, ApJ, 896, 37 Francis, L., Johnstone, D., Herczeg, G., et al. 2020, AJ, 160, 270 Frerking, M. A., Langer, W. D., \& Wilson, R. W. 1982, ApJ, 262, 590 Gaczkowski, B., Preibisch, T., Stanke, T., et al. 2015, A\&A, 584, A36 Gaudel, M., Maury, A. J., Belloche, A., et al. 2020, A\&A, 637, A92 Gueth, F., Guilloteau, S., \& Bachiller, R. 1996, A\&A, 307, 891 Gusdorf, A., Riquelme, D., Anderl, S., et al. 2015, A\&A, 575, A98 Hara, C., Kawabe, R., Nakamura, F., et al. 2020, arXiv:2010.06825 Hirano, N., Ho, P. P. T., Liu, S.-Y., et al. 2010, ApJ, 717, 58 Hirano, N., Liu, S.-Y., Shang, H., et al. 2006, ApJL, 636, L141 Hirano, S., Tsukamoto, Y., Basu, S., et al. 2020, ApJ, 898, 118 Hsieh, T.-H., Hirano, N., Belloche, A., et al. 2019, ApJ, 871, 100 Hsieh, T.-H., Murillo, N. M., Belloche, A., et al. 2018, ApJ, 854, 15 Inutsuka, S. 2012, PTEP, 2012, 01A307

Jiménez-Serra, I., Caselli, P., Martín-Pintado, J., et al. 2008, A\&A, 482, 549 Jiménez-Serra, I., Martín-Pintado, J., Caselli, P., et al. 2009, ApJ, 695, 149 
Jiménez-Serra, I., Martín-Pintado, J., Rodríguez-Franco, A., et al. 2004, ApJL, 603, L49

Jiménez-Serra, I., Martín-Pintado, J., Rodríguez-Franco, A., et al. 2005, ApJL, 627, L121

Jørgensen, J. K., Visser, R., Sakai, N., et al. 2013, ApJL, 779, L22

Klapper, G., Lewen, F., Gendriesch, R., et al. 2001, ZNatA, 56, 329

Klaus, T., Saleck, A. H., Belov, S. P., et al. 1996, JMoSp, 180, 197

Kristensen, L. E., van Dishoeck, E. F., Bergin, E. A., et al. 2012, A\&A, 542, A8

Larson, R. B. 1969, MNRAS, 145, 271

Lee, K. I., Dunham, M. M., Myers, P. C., et al. 2015, ApJ, 814, 114

Lee, C.-F., Stone, J. M., Ostriker, E. C., et al. 2001, ApJ, 557, 429

Lefloch, B., Castets, A., Cernicharo, J., et al. 1998, ApJL, 504, L109

Lim, J., Hanawa, T., Yeung, P. K. H., et al. 2016, ApJ, 831, 90

Lique, F., Dubernet, M.-L., Spielfiedel, A., et al. 2006, A\&A, 450, 399

López-Sepulcre, A., Watanabe, Y., Sakai, N., et al. 2016, ApJ, 822, 85

Machida, M. N., \& Basu, S. 2019, ApJ, 876, 149

Machida, M. N., Hirano, S., \& Kitta, H. 2020, MNRAS, 491, 2180

Machida, M. N., Inutsuka, S. I., \& Matsumoto, T. 2008, ApJ, 676, 1088

Masunaga, H., Miyama, S. M., \& Inutsuka, S.-i. 1998, ApJ, 495, 346

Matsumoto, T., Machida, M. N., \& Inutsuka, S. 2017, ApJ, 839, 69

Matsumoto, T., \& Tomisaka, K. 2004, ApJ, 616, 266

Maureira, M. J., Pineda, J. E., Segura-Cox, D. M., et al. 2020, ApJ, 897, 59

McMullin, J. P., Waters, B., Schiebel, D., et al. 2007, adass XVI, 376, 127

Mikami, H., Umemoto, T., Yamamoto, S., et al. 1992, ApJL, 392, L87

Misugi, Y., Inutsuka, S., \& Arzoumanian, D. 2019, ApJ, 881, 11

Mowat, C., Hatchell, J., Rumble, D., et al. 2017, MNRAS, 467, 812

Müller, H. S. P., \& Lewen, F. 2017, JMoSp, 331, 28

Müller, H. S. P., Spezzano, S., Bizzocchi, L., et al. 2013, JPCA, 117, 13843

Okoda, Y., Oya, Y., Sakai, N., et al. 2018, ApJL, 864, L25

Okoda, Y., Oya, Y., Sakai, N., et al. 2020, ApJ, 900, 40

Oya, Y., Sakai, N., Sakai, T., et al. 2014, ApJ, 795, 152

Pineda, J. E., Segura-Cox, D., Caselli, P., et al. 2020, NatAs, 4, 1158

Podio, L., Codella, C., Lefloch, B., et al. 2017, MNRAS, 470, L16

Punanova, A., Caselli, P., Feng, S., et al. 2018, ApJ, 855, 112

Rabli, D., \& Flower, D. R. 2010, MNRAS, 406, 95

Redaelli, E., Alves, F. O., Santos, F. P., et al. 2019, A\&A, 631, A154
Reipurth, B., Yu, K. C., Heathcote, S., et al. 2000, AJ, 120, 1449

Rygl, K. L. J., Benedettini, M., Schisano, E., et al. 2013, A\&A, 549, L1

Saigo, K., \& Tomisaka, K. 2006, ApJ, 645, 381

Sakai, N., Hanawa, T., Zhang, Y., et al. 2019, Natur, 565, 206

Sakai, N., Sakai, T., Hirota, T., Burton, M., \& Yamamoto, S. 2009, ApJ, 697, 769

Sakai, T., Sakai, N., Foster, J. B., et al. 2013, ApJL, 775, L31

Smith, M. D., Suttner, G., \& Yorke, H. W. 1997, A\&A, 323, 223

Spezzano, S., Caselli, P., Bizzocchi, L., et al. 2017, A\&A, 606, A82

Tachihara, K., Dobashi, K., Mizuno, A., Ogawa, H., \& Fukui, Y. 1996, PASJ, 48, 489

Tobin, J. J., Bourke, T. L., Mader, S., et al. 2019, ApJ, 870, 81

Tobin, J. J., Dunham, M. M., Looney, L. W., et al. 2015, ApJ, 798, 61

Tobin, J. J., Kratter, K. M., Persson, M. V., et al. 2016a, Natur, 538, 483

Tobin, J. J., Looney, L. W., Li, Z.-Y., et al. 2016b, ApJ, 818, 73

Tobin, J. J., Looney, L. W., Li, Z.-Y., et al. 2018, ApJ, 867, 43

Tokuda, K., Onishi, T., Saigo, K., et al. 2014, ApJL, 789, L4

Tothill, N. F. H., Löhr, A., Parshley, S. C., et al. 2009, ApJS, 185, 98

Tsukamoto, Y. 2016, PASA, 33, e010

van der Tak, F. F. S., Black, J. H., Schöier, F. L., et al. 2007, A\&A, 468, 627

van der Wiel, M. H. D., Jacobsen, S. K., Jørgensen, J. K., et al. 2019, A\&A, 626, A93

van Kempen, T. A., van Dishoeck, E. F., Hogerheijde, M. R., \& Güsten, R. 2009, A\&A, 508, 259

Velusamy, T., Langer, W. D., \& Thompson, T. 2014, ApJ, 783, 6

Wakelam, V., Ceccarelli, C., Castets, A., et al. 2005, A\&A, 437, 149

Watanabe, Y., Sakai, N., López-Sepulcre, A., et al. 2017, ApJ, 847, 108

Wiesenfeld, L., \& Faure, A. 2013, MNRAS, 432, 2573

Wurster, J., \& Li, Z.-Y. 2018, FrASS, 5, 39

Xu, L.-H., Fisher, J., Lees, R. M., et al. 2008, JMoSp, 251, 305

Yang, B., Stancil, P. C., Balakrishnan, N., et al. 2010, ApJ, 718, 1062

Yen, H.-W., Koch, P. M., Takakuwa, S., et al. 2017, ApJ, 834, 178

Yıldız, U. A., Kristensen, L. E., van Dishoeck, E. F., et al. 2015, A\&A, 576, A109

Zapata, L. A., Fernández-López, M., Rodríguez, L. F., et al. 2018, AJ, 156, 239

Zhang, Y., Tan, J. C., Tanaka, K. E. I., et al. 2019, NatAs, 3, 517

Zhao, B., Caselli, P., Li, Z.-Y., et al. 2018, MNRAS, 473, 4868 